\title{
History of Atomic Clocks
}

\author{
Norman F. Ramsey \\ Mount Holyoke College, South Hadley, MA 01075 \\ and Harvard University, Cambridge, MA 02138
}

Accepted: June 21, 1983

\begin{abstract}
The history of atomic and molecular standards of time and frequency is traced from the earliest work on molecular and atomic beam resonance techniques to more recent developments that promise improved standards in the future. The various devices currently used as standards are discussed in detail from an historical prospective. The latter part of the article is devoted to a discussion of prospective developments which hold promise for major improvements in accuracy, stability and reproducibility.
\end{abstract}

Key words: accuracy; atomic beam; clocks; frequency; frequency stabilization; laser; magnetic resonance; maser; microwave absorption; molecular bearn; optical pumping; reproducibility; stability; superconducting cavities; time; trapped-ion devices.

\section{Introduction}

In discussing the history of atomic and molecular standards of time and frequency, two alternative approaches are available. One is to treat all devices in parallel on a year-by-year basis. The other is to discuss each alternative device in succession. It is clear that the latter approach is the more suitable and will be followed here, but frequent cross-references will be made to other devices. In following this procedure, it is clear that the first technique discussed should be the molecular and atomic beam magnetic resonance method; historically, this was the first method and it remains one of the most effective time standards.

\section{The Molecular Beam Resonance Method}

The earliest molecular beam experiment was that of Dunoyer $[1]^{1}$ to show that $\mathrm{Na}$ atoms travel in straight lines in an evacuated tube. The development of molecular beams as a valuable research technique was largely due to the work of Otto Stern [2] and his collaborators in Hamburg in the 1920's and early 1930's, and to contributions from I. I. Rabi and his associates at Columbia in the 1930's.

The early molecular beam experiments did not use oscillatory fields and were of limited accuracy. The molecular beam magnetic resonance method arose from a succession of ideas, the earliest of which can be

About the Author, Paper: Higgins Professor of Physics at Harvard University, Norman F. Ramsey is one of America's foremost scientists in the precision measurement of the basic properties of matter. This invited paper is an outgrowth of the Fourteenth Annual Precise Time and Time-Interval meeting held at the NASA Goddard Space Flight Center on Dec. 2, 1982, at which a panel of distinguished scientists, including I. I. Rabi, H. G. Dehmelt, R. H. Dicke, R. V. Pound and C. H. Townes, as well as Professor Ramsey, discussed "An Historical Context for the Scientific Principles of Atomic Clocks." The same day marked the opening of a new exhibit on "Atomic Clocks" at the Smithsonian National Museum of American History. This paper was written by Professor Ramsey in response to an invitation of the committee that coordinated these events; it is based on an article by the author in the report, Science, Technology, and the Modern Navy, issued in 1976 by the U.S. Office of Naval Research in Arlington, VA as report ONR-37. An earlier review by the author appeared in IEEE Transactions on Instrumentation and Measurement IM-21, 90 (1972), and a more recent review by H. Hellwig, K. M. Evenson and D. J. Wineland appeared in Physics Today 31, 23 (December 1978).

\footnotetext{
${ }^{1}$ Numbers in brackets indicate literature references at the end of this paper.
} 
traced back to 1927 , although that idea was rather remote from the principle of magnetic resonance. Physicist Sir Charles Darwin [3]-the grandson of the great evolutionist-discussed theoretically the nonadiabatic transitions that make it possible for an atom's angular momentum components along the direction of a magnetic field to be integral multiples of $h / 2 \pi$ both before and after the direction of the field is changed an arbitrary amount. Inspired by Darwin's theoretical discussion, Phipps and Stern [4] in 1931 performed the first experiments on paramagnetic atoms passing through weak magnetic fields whose directions varied rapidly in space. Guttinger [5] and Majorana [6] developed further the theory of such experiments. Frisch and Segre [7] continued atomic beam experiments with adiabatic and nonadiabatic transitions of paramagnetic atoms and found, in agreement with Guttinger's and Majorana's theories, that transitions took place when the rate of change of the direction of the field was larger than or comparable to the Larmor frequency,

$$
\omega_{0}=\gamma_{1} H_{0}
$$

which is the classical frequency of precession of a classical magnetized top with the same ratio $\gamma_{I}$ of magnetic moment to angular momentum. Transitions did not take place when the rate of change of the direction of $H$ was small compared to the Larmor frequency. However, some of the results of Frisch and Segre were not consistent with theoretical expectations. Rabi [8] pointed out that these discrepancies arose from the effects of the nuclear magnetic moments since some of the transitions were performed in such weak fields that strong or intermediate coupling between the nuclei and the electrons prevailed. The transitions in such circumstances were quite different from those for which the effects of the nuclear spins could be neglected. Rabi showed that the resuits of Frisch and Segre were consistent with expectations if the effects of the nuclei were included. Rabi also pointed out that such nonadiabatic transitions could be used to identify the states and hence to determine the signs of the nuclear magnetic moments. Motz and Rose [9], Rabi [10], and Schwinger [11] in 1937 calculated the transition probability for molecules that passed through a region in which the direction of the field varied rapidly.

In all of the above experiments, however, the direction of the field varied in space, the only time variation arising as the atoms in the atomic beam passed through the region. Since the atoms possessed a Maxwellian velocity distribution, the atomic velocities varied and the apparent frequencies of the changing field were different for different velocities. Furthermore, the change in field direction ordinarily went through only a portion of a full cycle. For both of these reasons, no sharp resonance effects could be expected. Rabi in his brilliant 1937 theoretical paper entitled "Space Quantization in a Gyrating Magnetic Field" [10] assumed for simplicity that the field was oscillatory in time even though the primary application was to a field varying with the position along the beam rather. oscillating with time. As a consequence, all the formulae in that paper are applicable to the resonance case with oscillatory fields and the paper, without alteration, provides the fundamental theory for present molecular beam magnetic resonance experiments as well as for other experiments with magnetic resonance.

While writing his theoretical paper on the gyrating field, Rabi discussed with some of his colleagues the possibility of using oscillatory rather than space varying magnetic fields, but Rabi's laboratory had a full program of important experiments which did not require oscillatory fields, and no experiments utilizing oscillatory fields were started during the six months following the submission of Rabi's theoretical paper on the gyrating field. In September of 1937, C. J. Gorter visited Rabi's laboratory [12] and described his brilliantly conceived but experimentally unsuccessful efforts to observe nuclear magnetic resonance in lithium fluoride, as described in Gorter's publications of the previous year [13]. The research efforts in Rabi's laboratory were soon directed primarily toward the construction of molecular beam magnetic resonance apparatus with oscillator driven magnetic fields. Two successful molecular beam devices using this method were soon constructed by Rabi $[14,15]$, Zacharias [14,15], Millman [14], Kusch [14], Kellogg [15] and Ramsey [15]. A schematic view of these [14] is shown in figure 1 . In these experiments the atoms and molecules were deflected by a first inhomogeneous magnetic field and refocused by a second one. When a resonance transition was induced in the region between the two inhomogeneous fields, the occurrence of the transition could easily be recognized by the reduction of intensity asociated with the accompanying failure of refocussing. For transitions induced by the radio-frequency field, the apparent frequency was almost the same for all molecules independent of molecular velocity. As a result, when eq (1) was satisfied sharp resonances were obtained 


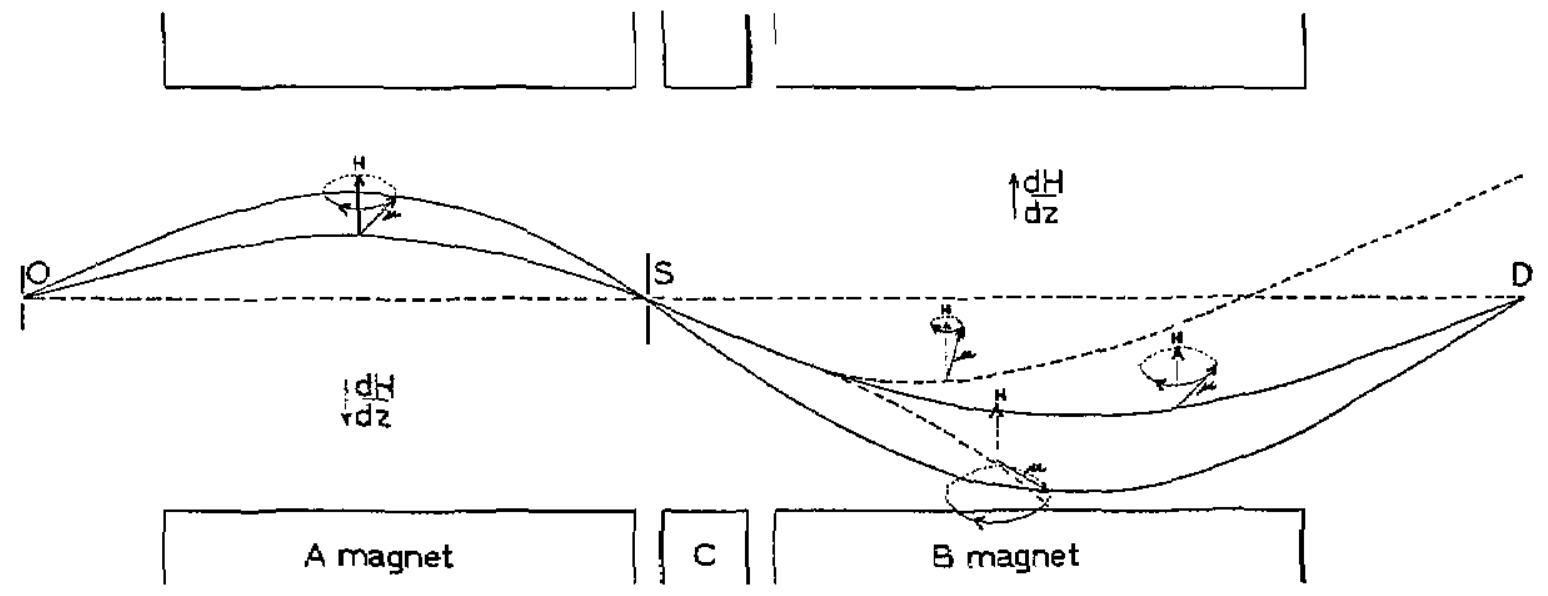

Fignre 1-Schematic diagram [12] showing the prineiple of the first molecular beam resonance apparatus. The two solid curves indicate two paths of molecules having different orientations that are not changed during passage through the apparatus. The two dashed curves in the region of the $B$ magnet indicate two paths of molecules whose orientation has been changed in the $C$ region so the refocusing is lost due to the change in the component of the magnetic moment along the direction of the magnetic field.

Rabi et al. [15] soon extended the method to the molecule $\mathrm{H}_{2}$, for which the resonance frequencies depended not only on eq (l) but also on internal interactions within the molecule. The transitions in this case occurred whenever the oscillatory field was at a Bohr frequency for an allowed transition

$$
h \nu=E_{1}-E_{2}
$$

For the first time these authors began speaking of their results as "radio-frequency spectroscopy."

\section{Molecular Beam Magnetic Resonance Experiments}

By 1939 the new molecular beam magnetic resonance method had demonstrated its usefulness sufficiently well that it appeared to Rabi and his associates to be of possible value for the definition of standard magnetic fields and for use as a time and frequency standard; these potentialities were discussed informally with some scientists at the National Bureau of Standards in 1939. Rabi [16] in his 1945 Richtmeyer Lecture publicly discussed the possibility of atomic clocks and expanded on the implications in a subsequently reported New York Times interview.

In most respects the molecular beam technique in 1939 was more suitable as a standard of magnetic field than of frequency or time since the observed resonances then were largely dependent on the externally applied magnetic field. From the point of view of atomic clocks, it was consequently a great step forward when in 1940 Kusch, Millman, and Rabi $[17,18]$ first extended the method to paramagnetic atoms and in particular to $\Delta F= \pm 1$ transitions of atoms where the relative orientation of the nuclear and electronic magnetic moments was changed, in which case the resonance frequencies were determined dominantly by fixed internal properties of the atom rather than by interactions with an externally applied magnetic field. The first resonance measurements of the Cs hyperfine separation, which has been so extensively used in frequency control, was reported [18] in 1940.

In 1941 the research with the atomic beam magnetic resonance method was mostly interrupted by World War II and did not resume until 1946. In 1949 Kusch and Taub [19] pointed out the possibility of observing the hyperfine resonances at magnetic fields such that the resonance frequency was an extremum, in which case the frequency to first order was independent of the strength of the magnetic field.

In 1949 Ramsey [20,21] invented the separated oscillatory field method for a molecular beam resonance experiment on molecular hydrogen. In this new method the oscillatory field, instead of being distributed uniformly throughout the transition region, was concentrated in two coherently driven oscillatory fields in short regions at the beginning and end of the transition region. The theoretical shape of a resonance curve with this apparatus is shown in figure 2 . Ramsey pointed out that this method has the following advantages: (1) the resonances are $40 \%$ narrower than even the most favorable Rabi resonances with the 


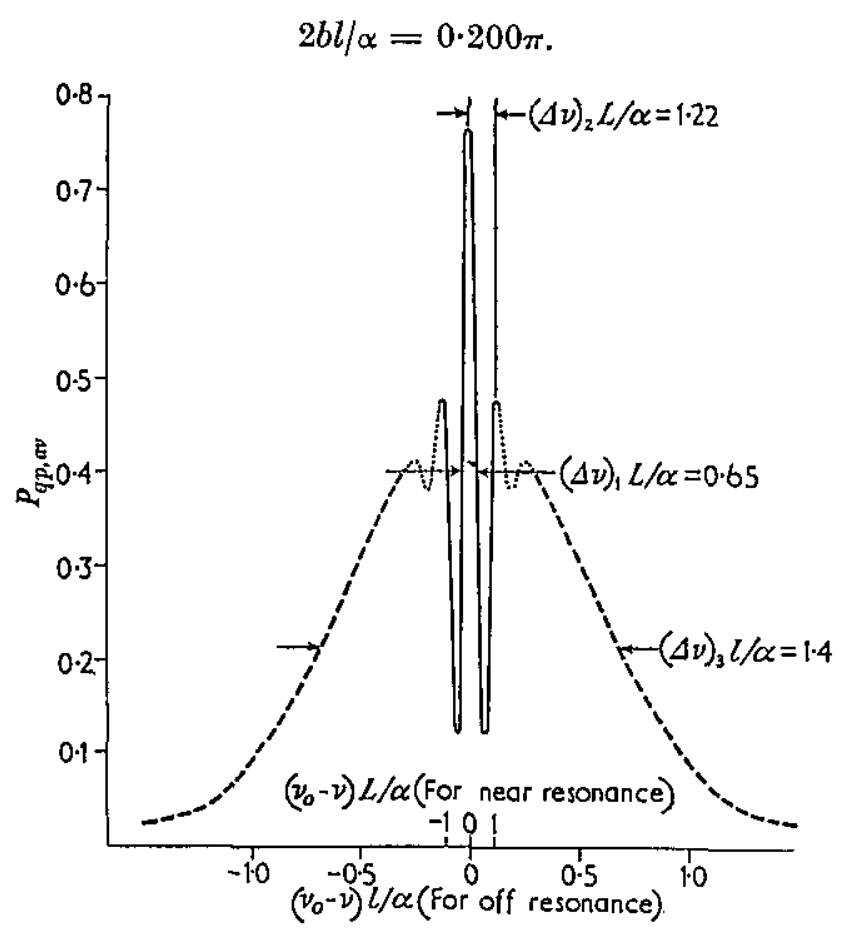

Figure 2-Theoretical shape for separated oscillatory field resonance pattern [20].

same length of apparatus; (2) the resonances are not broadened by field inhomogeneities; (3) the length of the transition region can be much longer than the wavelength of the radiation, provided that the two oscillatory regions are short, whereas there are difficulties with the Rabi method due to phase shifts when the length of the oscillatory region is comparable to the wavelength; (4) the first-order Doppler shift can mostly be eliminated when sufficiently short oscillatory field regions are used; (5) the sensitivity of resonance measurements can be increased by the deliberate use of appropriate relative phase shifts between the two oscillatory fields; (6) with short-lived states the resonance width can be narrowed below that expected from the lifetime of the state and the Heisenberg uncertainty principle if the separation of the oscillatory fields is sufficiently great that only molecules living longer than average in the excited state can reach the second oscillatory field before decaying [22]. The apparatus for which the separated oscillatory field was first proposed is shown in figure $3[23]$.

\section{Atomic Beam Frequency Standards}

With the above developments, it was apparent to most molecular beam researchers that atomic beam methods could be highly effective for standards of time and frequency even though this view was not shared by advocates of alternative frequency standards, such as crystals. In 1952, Sherwood, Lyons, McCracken and Kusch [24,25] reported briefly on atomic beam resonance research supported by the National Bureau of Standards and directed primarily toward the development of an atomic beam clock. A schematic diagram of a proposed atomic beam clock at that time is given in figure 4. The financial support for such work soon dwindled due to advances in the then new field of microwave spectroscopy and to the view then held at the National Bureau of Standards that a molecular clock based on the microwave absorption by ammonia at its inversion frequency would be simpler and more promising.

A few years later, Zacharias [26,27] stimulated renewed interest in an atomic beam cesium clock. His initial concern was for an entirely new type of cesium beam in which ultrahigh precision would be obtained by the use of extremely slow molecules moving upwards in a vertical apparatus at such low velocities that they would fall back down by the action of gravity. Although this fountain experiment eventually failed due to the unexpected deficiency of the required ultraslow molecules emerging from the source, it stimulated Zacharias to develop and to urge others to develop well-engineered atomic beam frequency standards using normal atomic velocities. The unsuccessful fountain experiment of Zacharias illustrates the value to science even of some unsuccessful experiments; this unsuccessful effort directly and indirectly stimulated three quite different but important developments: (1) the use of conventional but well-engineered atomic beams for frequency control; (2) the development by Kleppner, Ramsey, and others [28-30] of the stored-atom technique, which eventually led to the hydrogen maser; and (3) high-precision resonance experiments with ultraslow neutrons [31]. The first report on an atomic beam frequency standard at a Frequency Control Symposium was that of Zacharias in 1955. Zacharias claimed a short-time stability of 1 part in $10^{9}$ for his atomic cesium frequency standard.

In 1955 Essen and Parry [32] of the British National Physical Laboratory successfully operated the first practical laboratory atomic cesium beam apparatus that was extensively used as an actual frequency standard. Their construction and effective use of this device provided a major impetus to the subsequent development of atomic beam cesium frequency standards.

In 1956 the first commercial model of an atomic beam frequency standard appeared on the market. 


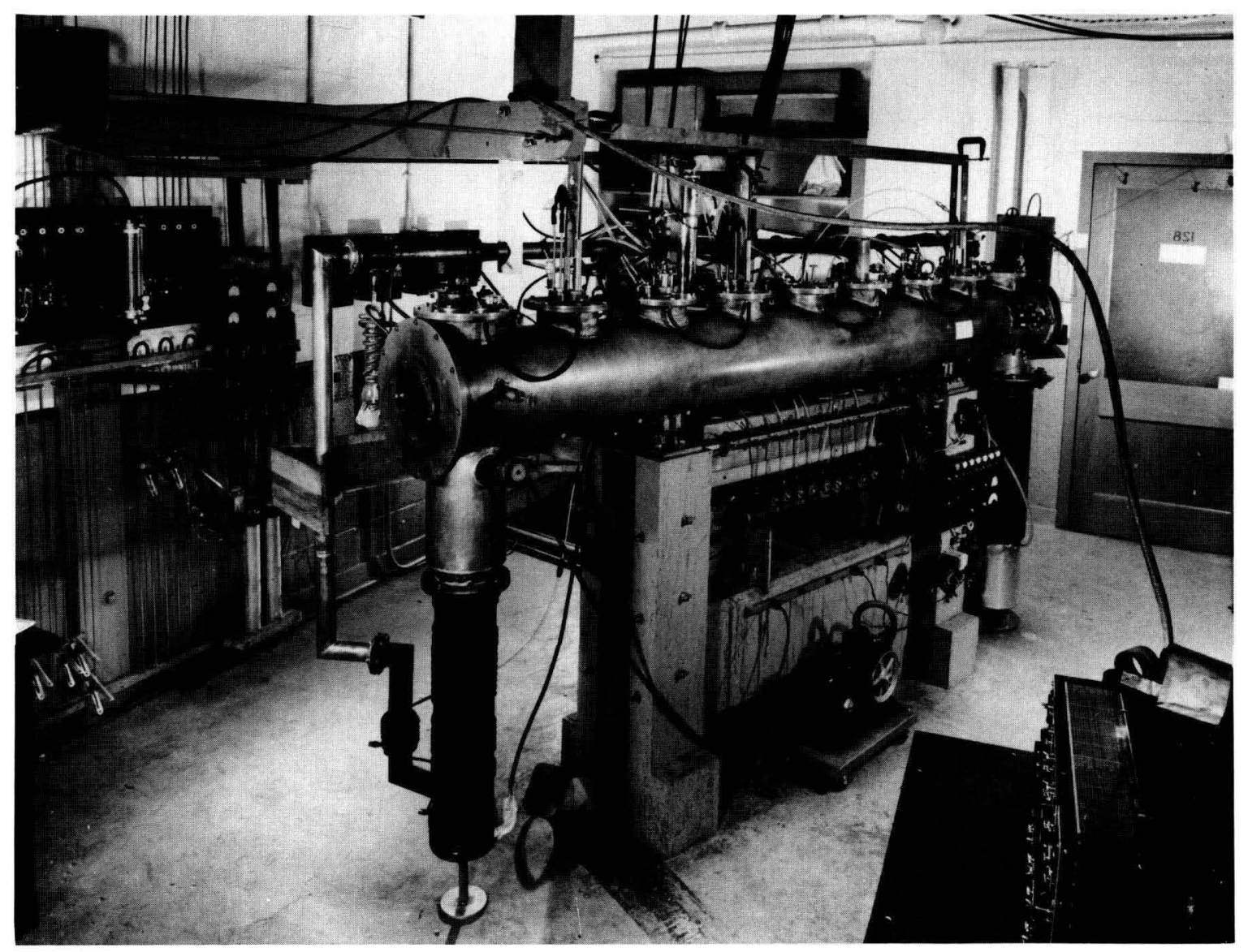

Figure 3-Apparatus for which separated oscillatory field was first proposed [18].

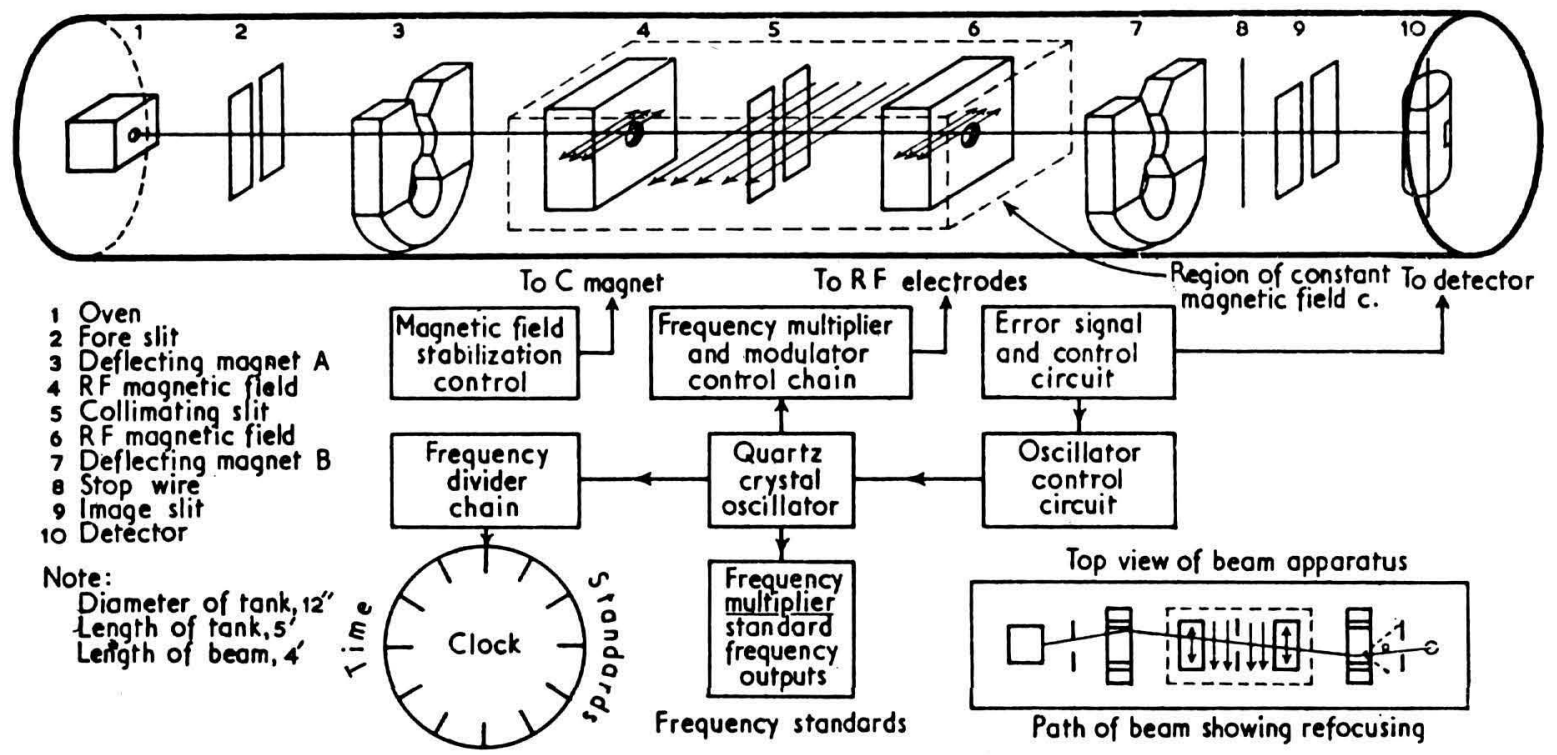

Figure 4-Schematic diagram of a proposed atomic beam clock [25]. 

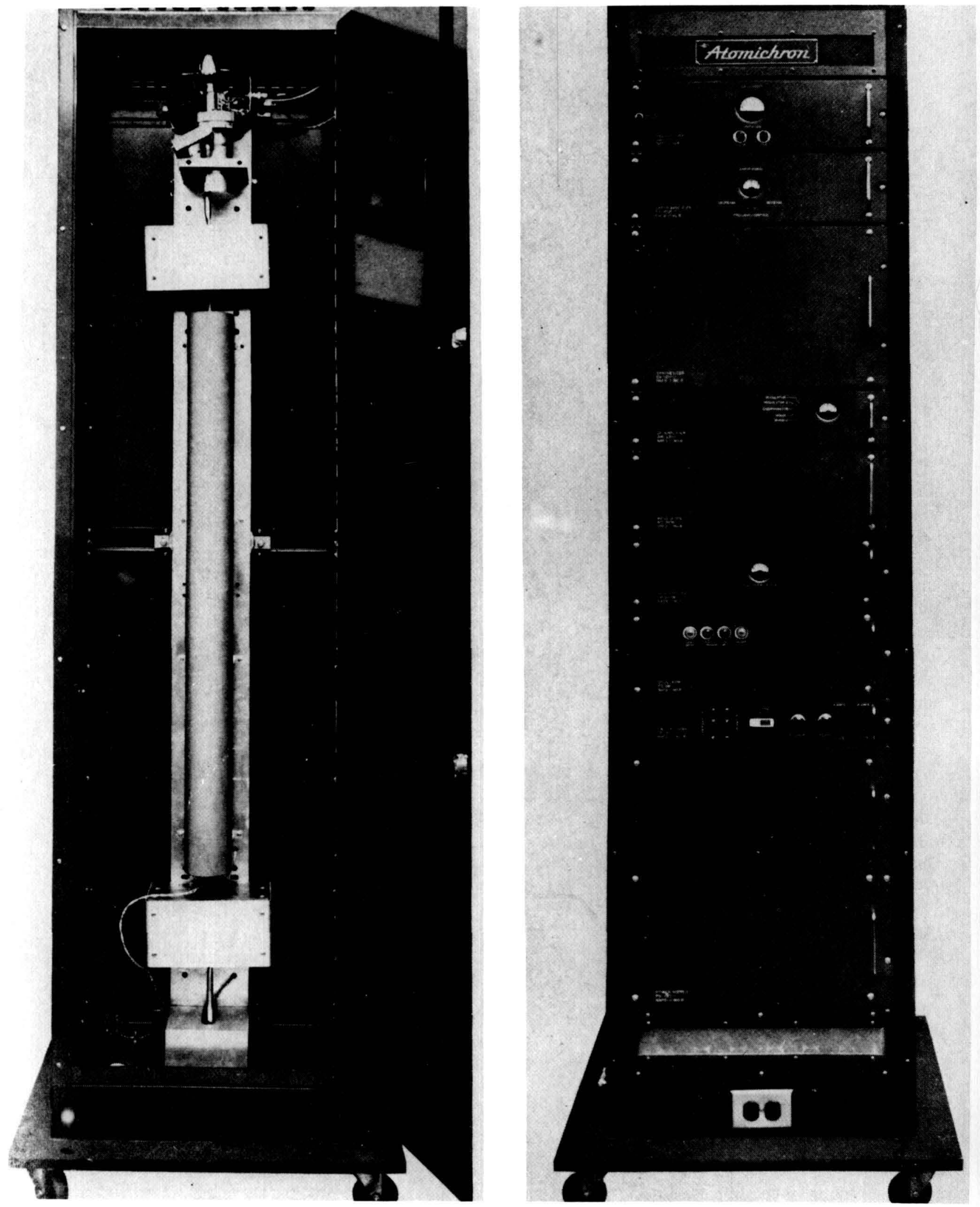

Figure 5-First commercial atomic beam frequency standard. National's Atomichron [33]. (C) 1972 by the IEEE. 
This was National's Atomichron developed [33] by Daly and Orenberg in collaboration with Zacharias and further improved by Holloway and McCoubrey. This device used Ramsey's separated oscillatory field method for increased precision, a special design of cesium oven that could be operated several years without exhaustion, titanium pumping to permit permanent sealing off of the evacuated beam tube, and many other features generally necessary for an effective commercial device. The first commercial Atomichron is shown in figure 5. The development of the Atomichron was supported financially largely by the U.S. Signal Corps at Ft. Monmouth, NJ, and the Office of Naval Research, although some support came from the Air Force. A purchase order by the Signal Corps for a relatively large number of Atomichrons made possible the development of massproduction techniques and improved engineering to permit sufficient reliability and reductions in price to assure commercial success.

The early atomic beam frequency standards were subject to various frequency shifts dependent on the amplitude of the radio-frequency power and on other variables. To account for these results, Ramsey, with the aid of computational analysis supported by the National Co., investigated the various possible distortions that would occur in an atomic beam resonance [34]. The elimination of radio-frequency phase shifts and other sources of distortion made possible the marked increases in accuracy that have been obtained with the atomic beam frequency standards.

From 1956 on, atomic beam frequency standards developed rapidly. Mockler, Beehler, Barnes, Hellwig, Wineland and others [33,35,36] developed an atomic cesium frequency standard at the National Bureau of Standards in Boulder, CO. Other commercial organizations such as TRG, Bomac, Varian, HewlettPackard, Frequency and Time Systems, Inc., and Frequency Electronics, Inc. became involved. Many laboratories throughout the world either constructed or purchased atomic beam frequency standards including those in Canada, France, Germany, USSR, China and the laboratories of Kartaschoff [35] and Bonanomi [35] in Switzerland. Reder, Winkler and others [35] at Ft. Monmouth and Markowitz at the Naval Observatory sponsored various worldwide studies of the comparison of atomic clock frequencies and the synchronization of clocks. Extensive studies were made of other atoms such as thallium for use in the atomic veam tubes, and various molecular resonances were studied for possible use in a molecular beam electric resonance apparatus for frequency control purposes. A $\mathrm{Tl}^{205}$ frequency measurement accurate to 2 parts in $10^{11}$ was reported by Bonanomi [37]. However, atomic cesium remains the most widely used substance in molecular or atomic beam frequency control devices. Particularly effective atomic beam cesium clocks were developed and sold by Hewlett-Packard which also developed a "flying clock" particularly suitable for the intercomparison of atomic clocks in different laboratories. A typical beam tube for an atomic cesium frequency standard is shown in figure 6. Accuracies as high as 1 part in $10^{13}$ have been claimed for some laboratory cesium standards [35].

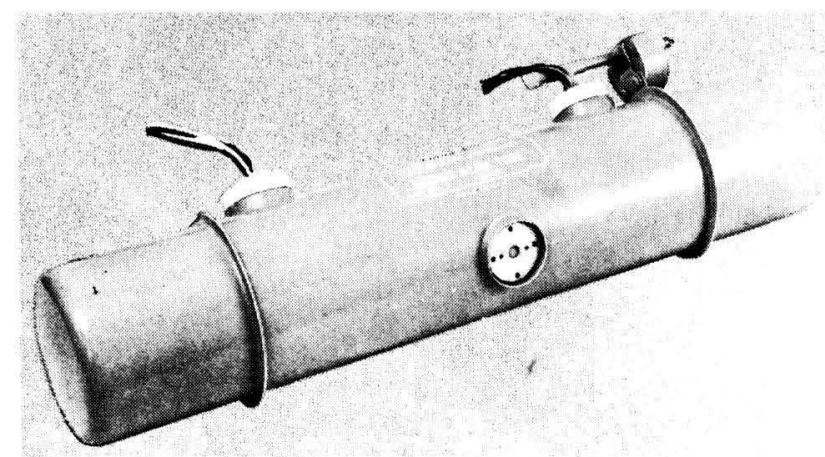

Figure 6-Beam tube for atomic cesium standard manufactured by Varian Associates for Hewlett-Packard [33]. (C) 1972 by the IEEE.

In 1967, the 13th General Conference on Weights and Measures resolved that the unit of time in the International System of Units should be the second defined as follows: "The second is the duration of 9192631770 periods of the radiation corresponding to the transition between the two hyperfine levels of the ground state of the cesium atom 133," a definition that is still retained.

\section{Microwave Absorption Spectroscopy}

Microwave absorption spectroscopy had an early start in the experiments of Cleeton and Williams $[38,39]$ who observed the absorption of microwave radiation at the $\mathrm{NH}_{3}$ inversion frequency. However, research on microwave absorption was inhibited at that time by the lack of suitable microwave oscillators and circuits so there was no further development of microwave absorption spectroscopy until after the development of microwave oscillators and waveguides for radar components in World War II. Immediately following World War II there was a great burst of activity in microwave absorption spectroscopy. Although there were no publications on experimental microwave spectroscopy in 1945 , in the single year of 
1946 there were a number of important publications from many different laboratories including reports by the following authors [40]: Bleaney, Penrose, Beringer, Townes, Dicke, Lamb, Becker, Autler, Strandberg, Dailey, Kyhl, Van Vleck, Wilson, Dakin, Good, Coles, Hershberger, Lamont, Watson, Roberts, Beers, Hill, Merritt, and Walter, and in 1947 there were more than 60 published papers on this subject including a number of publications by Gordy and Jen, by authors with reports the previous year, and by others. A typical microwave absorption experiment at this time is shown schematically in figure 7 .

Microwave absorption techniques were quickly recognized to be of potential value for frequency standards. In 1948 a group of workers [25] at the National Bureau of Standards built an ammonia clock that was completed in 1949 and is shown in figure 8. It eventually achieved an accuracy of 1 part in $10^{8}$. Rossell [25] in Switzerland and Shimoda in Japan devised an improved ammonia absorption clock good to a few parts in $10^{9}$.

In 1951 Townes [41] analyzed frequency stabilization of microwave spectral lines and the application to atomic clocks. In the references he lists other early contributors to the field. At the 1951 Fifth Frequency Control Symposium, Dicke [42] reported on microwave absorption molecular frequency standards. In the seventh, eighth and ninth symposia, Dicke, Carver, Arditi, and others described the continuation of this work at both Princeton and the
Radio Corporation of America (RCA) with the financial support of the Signal Corps and the Office of Naval Research [35,42]. The microwave absorption studies soon merged with the optical pumping techniques, described in the next section, since the intensities of the resonances were greatly enhanced by the use of optical pumping.

\section{Optical Pumping}

The starting point of all research on optical pumping was a paper by Bitter [43] in 1949, which showed the possibility of studying nuclear properties in optically excited states. Kastler $[44,45]$ showed the following year that this technique could be effectively combined with the double resonance method he and Brossel [44] had developed. Both optical pumping and optical detection techniques served the purpose of increasing the signal-to-noise ratio of the resonator output signal. The optical pumping greatly enhances the population of certain states so the signal is not weakened by stimulated emission nearly cancelling absorption and the optical detection increases the signal-to-noise ratio because of the lower noise level of optical detectors over microwave detectors.

The combination of optical pumping techniques with the buffer gas method for reducing Doppler shift developed by Dicke [33,42] provided gas cells of real value as frequency-control devices. Although many

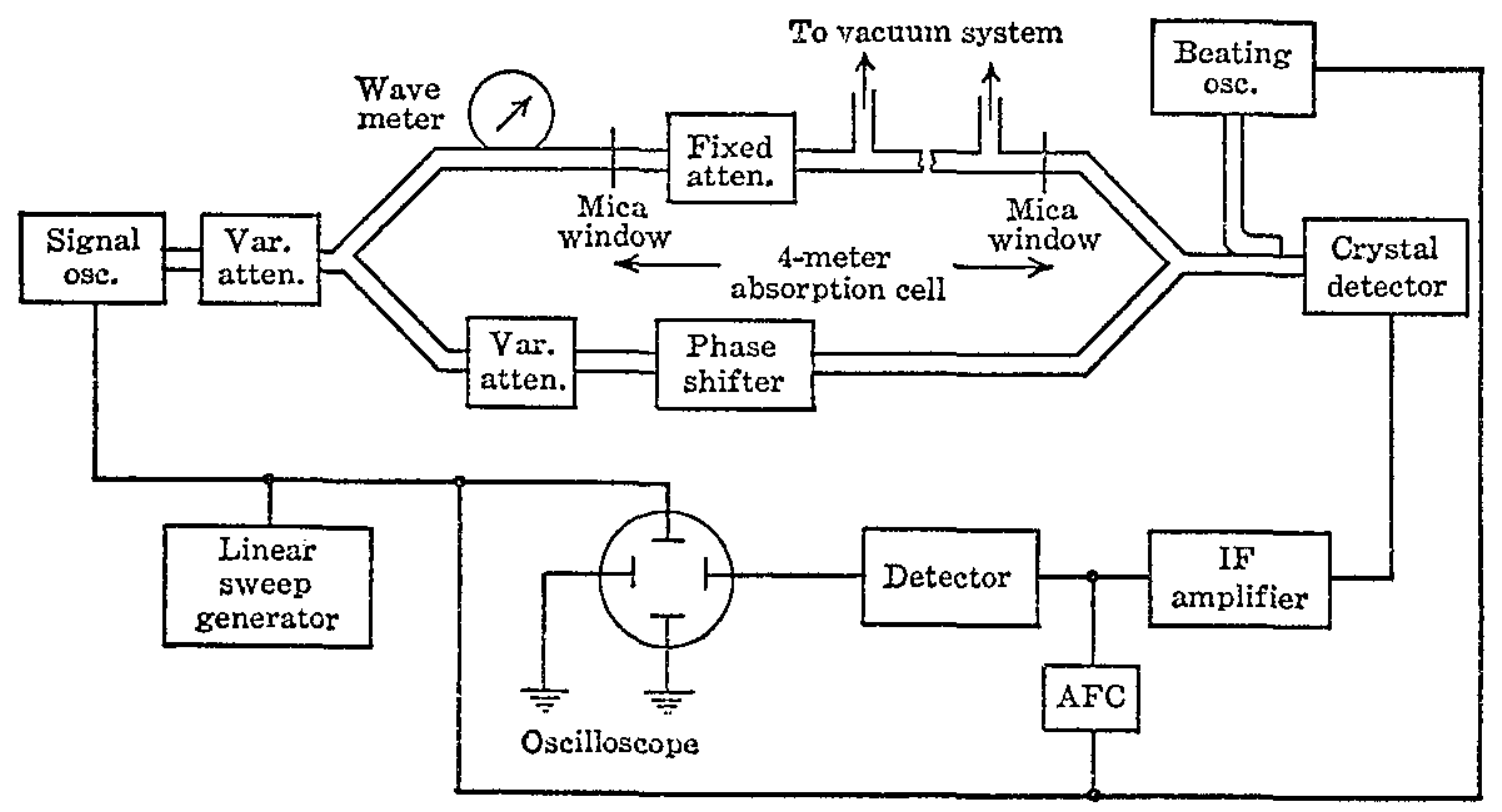

Figure 7-A typical microwave absorption experiment using a radio-frequency bridge and heterodyne detection. 


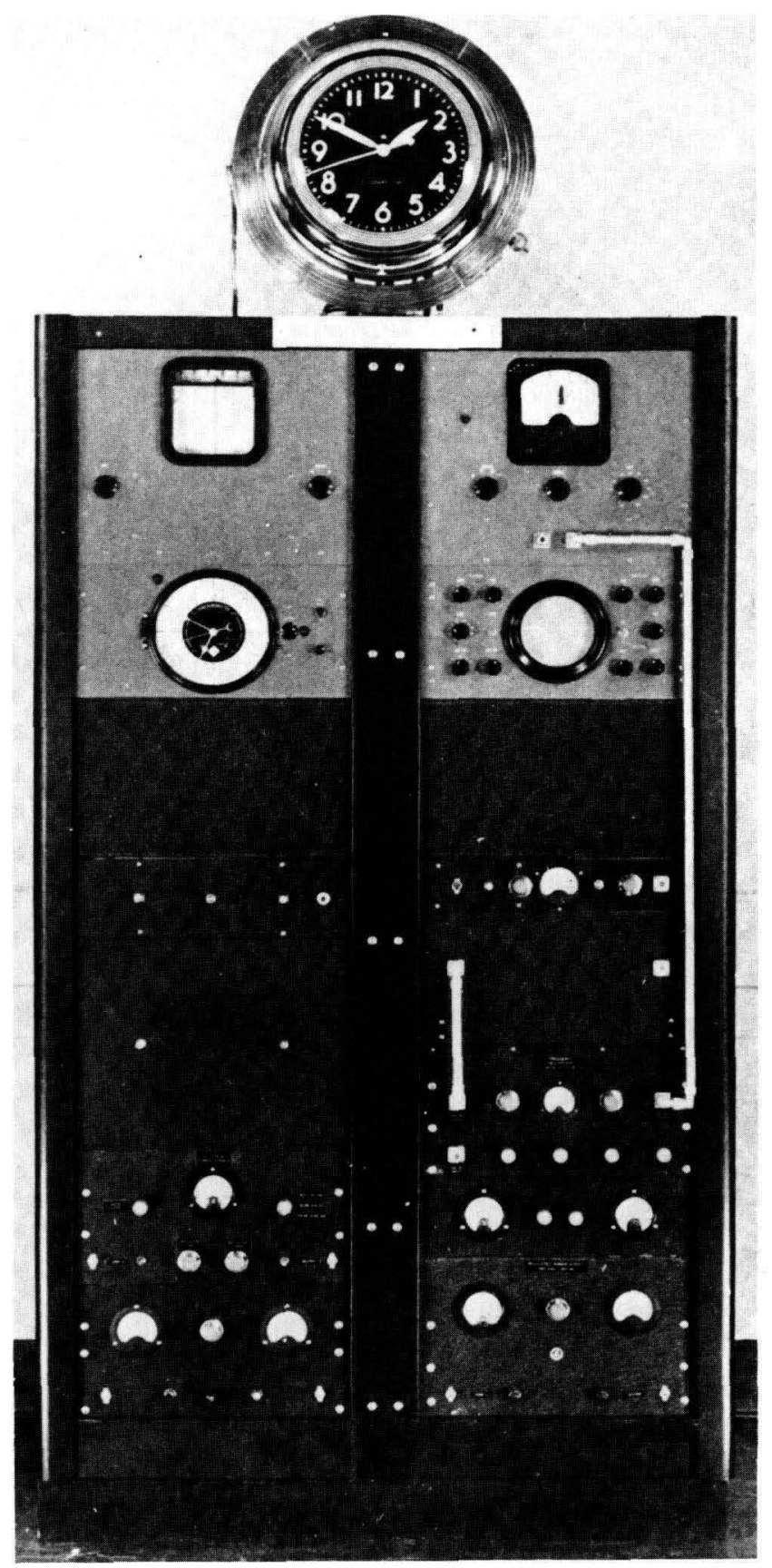

Figure 8-National Bureau of Standards ammonia clock [25]. (C) 1972 by the IEEE.

different atoms have been used in such gas cells, $\mathbf{R b}^{87}$ soon became the favorite in most such devices. Extensive work in optically pumped gas cells for frequency control has been done at Princeton, RCA, International Telephone \& Telegraph (ITT), Space Technology Laboratory, the National Bureau of Standards, Clauser Technology Corporation, Varian Associates, and many other commercial, university, and government organizations in the United States and abroad. Figure 9 shows a typical optically pumped rubidium frequency standard.

The optically pumped gas cells have the advantages of simplicity, relatively low cost, large signal-to-noise ratio, and good spectral purity. Unfortunately, the relatively large shift in frequency due to numerous buffer gas collisions is dependent on purity, pressure, and temperature. Changes in the light intensity shift due to variations in the pumping lamp intensity or spectrum may also be a problem. As a result, the stability of rubidium gas cells over a period of several months is ordinarily no better than a few parts in $10^{10}$. These shifts prevent the optically pumped gas cells from being primary time and frequency standards, but the gas cells are used as frequency control devices when their accuracy is sufficient. Research is currently in progress in a number of laboratories to improve the stability of optically pumped gas cells; Bouchiat, Brossel [46], and others, for example, have eliminated the buffer gases as was done earlier in the hydrogen maser [47] and have used suitable coated walls to retain the atoms and reduce the effect of the first-order Doppler shift.

\section{Molecular Masers}

In 1951, Pound, Purcell and Ramsey [48] studied nuclear spin systems with inverted populations and noted that such systems in principle were intrinsic amplifiers rather than absorbers. The first suggestions actually to use systems with inverted populations as practical amplifiers or oscillators were made independently in the early 1950's by Townes [49] and independently but somewhat later by Weber [50] and Basov and Prokhorov [27]. The first such amplifier was successfully constructed in 1954 by Gordon, Zeiger, and Townes [49] and called a maser (Microwave Amplifier by Stimulated Emission of Radiation). The device used inhomogeneous electric fields to focus the higher energy molecular inversion states of ammonia molecules in a molecular beam. These molecules then emitted coherent stimulated emission radiation in passing through a cavity tuned to the $24-\mathrm{GHz}$ ammonia inversion transition. A schematic diagram of the first ammonia maser is shown in figure 10. A report by Gordon on the new ammonia maser was a major attraction at the special meeting on atomic and molecular resonances sponsored by the Signal Corps Engineering Laboratory in 1956. In that year Bloembergen [29] proposed the three-level solidstate maser and in 1958 Townes and Schawlow [30] pointed out the possibility of masers at the infrared and optical frequencies. 


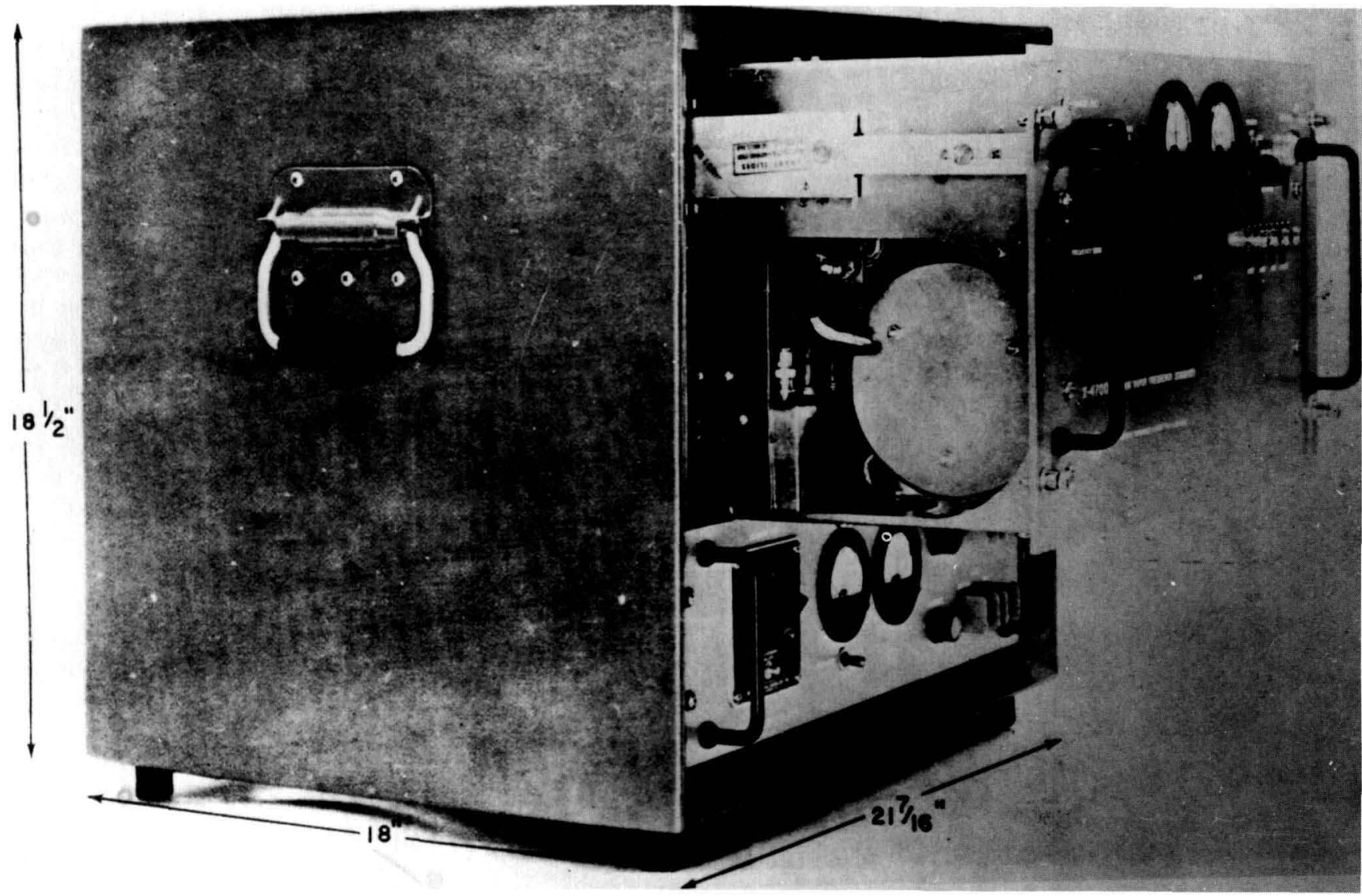

Figure 9-Rubidium frequency standard. (C) 1972 by the IEEE.

Since the announcement of the first successful ammonia maser in 1955 there has been tremendous research and development activity by scientists and engineers in many countries. Masers at infrared or optical frequencies (lasers) have great potential for frequency control. Further discussions of lasers will be
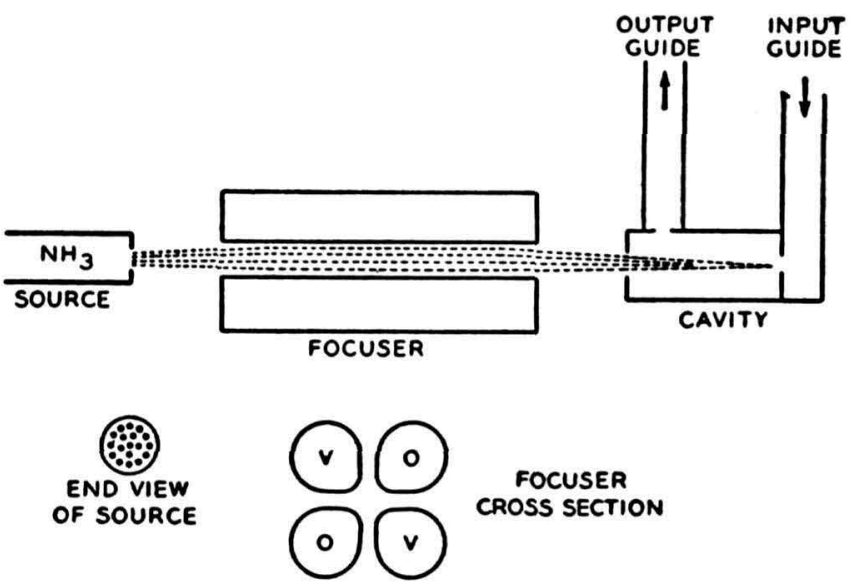

Figure 10-Schematic diagram of original ammonia maser [49]. deferred to a later section of this report. Molecular maser developments for the purposes of time and frequency control soon became intense and went in many directions including the search for more suitable molecules than ammonia, the development of two cavity masers analogous to the separated oscillatory field method [20] for molecular beams, the use of ammonia of different isotopic composition, and so forth. A value of the $\mathrm{N}^{15} \mathrm{H}_{3}$ frequency accurate to 5 parts in $10^{11}$ has been obtained by de Prins and confirmed by Barnes [35]. However, after a few years of intense molecular maser activity, the interest in such masers for time standards and frequency control waned since the molecular masers on the one hand lacked the simplicity and low cost of optically pumped rubidium gas cells and on the other hand lacked the high stability of either atomic cesium beams or atomic hydrogen masers.

\section{Atomic Masers}

In 1957 Ramsey [35] proposed to increase the accuracy of the atomic beam magnetic resonance method by retaining the atoms for a much longer time 
between the two separated oscillatory fields, thereby obtaining much narrower resonances. His first thought was to confine the atoms with inhomogeneous magnetic fields in a large ring. However, it soon became apparent that the inhomogeneous confining magnet fields, which acted on the atoms for long periods of time, would hopelessly broaden the resonances. In fact, it became clear that the frequencies would be much less perturbed by a confinement force that was present for only a short fraction of the time even though the force might be stronger when it was applied. The obvious limit of such a device was confinement of atoms in a box with suitably coated walls. Although many wall bounces would be required to achieve marked narrowing of the resonance by a long storage time, the first experiment involved only a few wall collisions, since most scientists at that time believed that even atoms in the $\mathbf{S}$ state would undergo hyperfine transitions at a single wall collision. The first experiment of Kteppner, Ramsey; and Fjektadt [51] involved only a few wall collisions and the experiment was appropriately called a "broken atomic beam resonance experiment." Cesium atoms and a Teflon-coated [52] wall were used in these first experiments.

Goldenberg, Kleppner, and Ramsey [53] then made an atomic beam resonance apparatus that stored atoms of cesium for a longer time and investigated alternate wall-coating material. They found that when the storage bulb was coated with a paraffin-like substance called Paraflint [54,53] resonances could be observed after as many as 200 wall collisions. It was recognized that atomic hydrogen would probably be a more suitable atom than cesium because of the low electric polarizability and the low mass of hydrogen, but cesium could be much more efficiently detected than hydrogen.

Kleppner and Ramsey [51,53,47] therefore proposed detection of the emitted radiation rather than of the atom. In particular, they noted that atoms of hydrogen in the higher energy hyperfine state could be focused into a suitably coated storage bulb by a six-pole magnet while acoms in the lower state would be defocused. They showed that if such a storage bulb were surrounded by a microwave cavity tuned to the $1420 \mathrm{MHz}$ hyperfine transition frequency, then maser oscillation should occur. In 1960, Goldenberg, Kleppner, and Ramsey [47] constructed and operated the first atomic hydrogen maser. This apparatus is shown in figure 11. Although the total microwave power was small-approximately $10^{-12}$ W-the stability was so high that the output was concentrated into an extremely narrow band with a consequently favorable signal-to-noise ratio.

Although the first hydrogen maser used a wall coating of Paraflint or of Dri-Film [55] (dimethyldichlorosilane [53]), it was soon found that with atomic hydrogen, in contrast to cesium, Tefloncoated [52] walls gave longer storage times and smaller frequency shifts from wall collisions [56]. Bender [57] soon pointed out that spin exchange collisions of hydrogen atoms could not be neglected and might produce a significant frequency shift, but Crampton [58] noted that the normal tuning technique would cancel out such an effect. Later Crampton [59] pointed out the existence of a smaller additional spin exchange effect that would not be cancelled by the normal tuning method. This effect was omitted in earlier theories due to their neglect of the contribution of the hyperfine interaction during the short duration of the collision. Crampton [59] developed a technique for measuring the spin exchange effect. Crampton also pointed out the existence of a small frequency shift [59] due to magnetic field inhomogeneities: this small shift is often called the Crampton eifect. Both of these effects are so small they did not affect past measurements and they can be further reduced by suitable apparatus design. Kleppner, Ramsey and their associates have developed and reviewed the theory and techniques for the atomic hydrogen maser $[56,60]$.

A commercial hydrogen maser [60] was developed by Vessot, Peters, Vanier, McCoubrey, Levine and Cutler. The work was started at Bomac and successively transferred to Varian Associates and Hewlett-Packard. The H-10 maser developed by Vessot and his associates is shown in figures 12 and 13 . Since then hydrogen masers have been developed and constructed at the Smithsonian Astrophysical Observatory by Vessot and his associates; at the Goddard Space Flight Center by Peters, Reinhardt, and others; in France by Audoin [61] and Grivet [35] in Canada by Vanier and by scientists at the NRC; in Switzerland by Kartaschoff [35]; at Harvard by Ramsey, Kleppner, Crampton, Myint, Brenner, Uzgiris, Zitzewitz, Debely, and Larson; at the National Bureau of Standards by Hellwig; at Williams College by Crampton; at the Jet Propulsion Laboratory; at the firm, $A S U A G$; at Hughes Aircraft by Wang; at Efratom; at the Applied Physics Laboratory; in the USSR at the Lebedev Institute and the Moscow State University; and in the Peoples Republic of China at Shanghai and Shianxi Observatory and at the National Institute of Metrology [62].

The hydrogen maser eliminates first-order Doppler shifts and photon recoil effects by virtue of the 


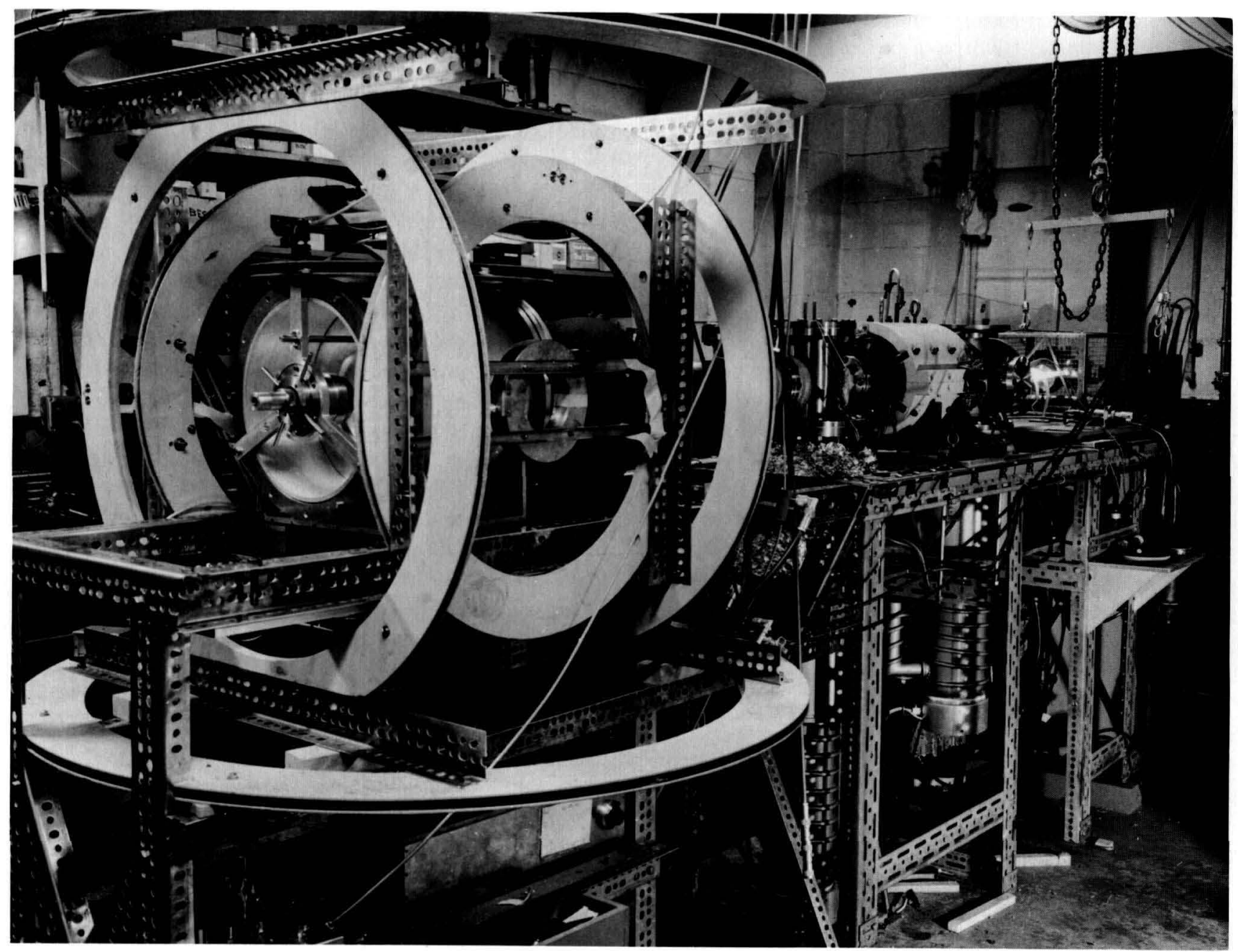

Figure 11-Original hydrogen maser [47]. The large coils are to cancel external magnetic fields. In later hydrogen masers, these were replaced by two or three concentric cylinders of highpermeability magnetic shielding.

Figure 12-Schematic diagram of a commercial hydrogen maser developed by Vessot and his associates [60].

confinement of the atoms in a box where the average velocity is essentially zero and by absorption of recoil momentum by the confining box. The hydrogen maser also benefits from the relatively long storage time with a resulting narrow resonance line and from the low noise characteristic of maser amplification. It shares with most other atomic or molecular frequency standards the need for correcting for the small secondorder Doppler shift.

The chief disadvantage of the hydrogen maser for time and frequency control has been the existence of a

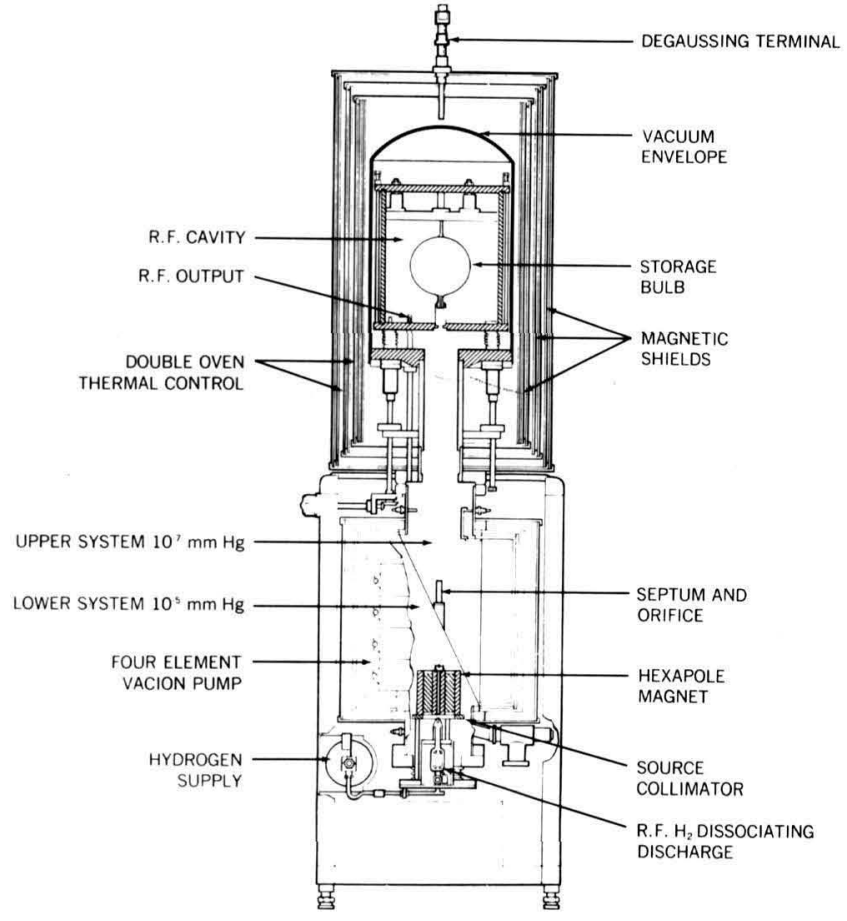




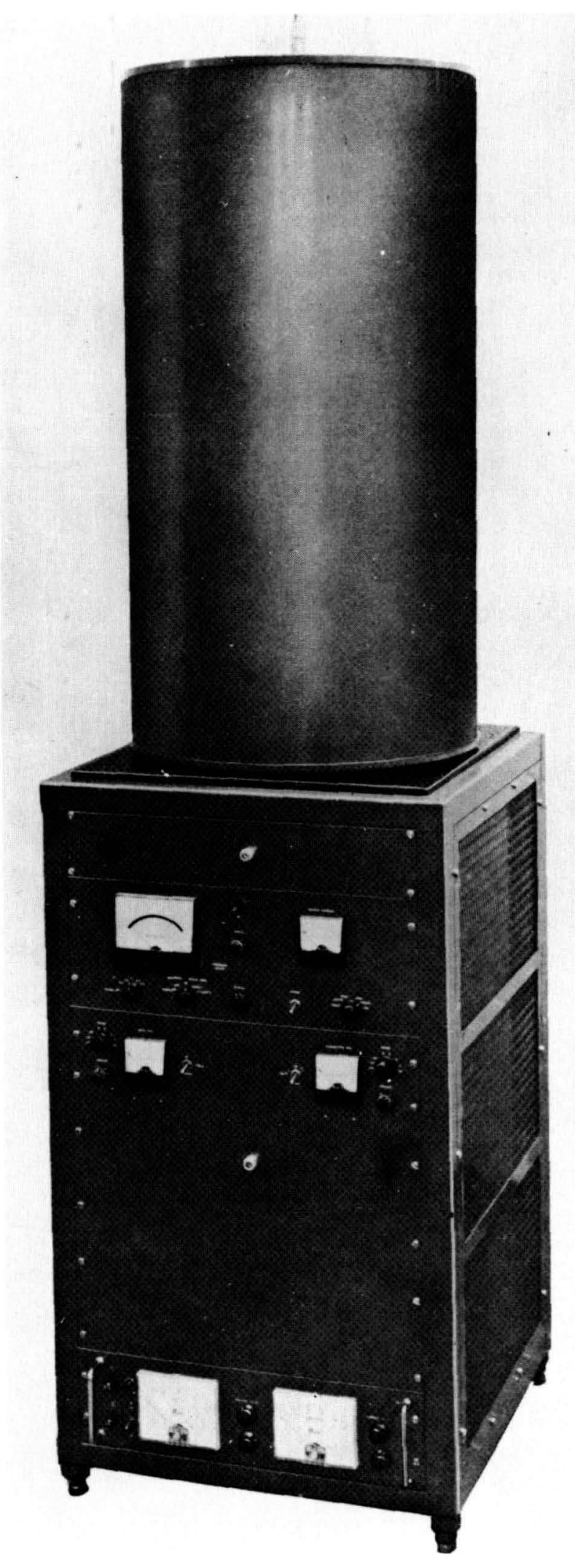

Figure 13-Commercial hydrogen maser [60]. (C) 1972 by the IEEE. small frequency shift due to collisions of the atoms with the Teflon-coated walls of the storage bulb. With a 16-cm-diameter bulb this wall shift is about 2 parts in $10^{11}$ and can be measured by using bulbs of two different diameters. Until recently the measurements of the wall shifts have been limited to accuracies of a few percent by variations in different wall coating. However, Uzgiris and Ramsey [63] at Harvard have reduced the wall shift by a factor of 10 by the use of an atom storage vessel 10 times larger in diameter (1.5 $\mu \mathrm{m})$. In the same laboratory, Brenner [64] and Debely [65] have developed a technique to measure the wall shift in a single storage bulb by making a change in the bulb's volume by deforming its shape. Since a single bulb is used in this method, it is free from the uncertainties in the nonreproducibility of the wall coatings of different bulbs. This method was first used on hydrogen masers with normal-size storage vessels, but Reinhardt [66] later applied it to a maser with a large storage vessel as well. Zitzewitz [67] has shown that at a temperature of about $80^{\circ} \mathrm{C}$ the wall shift passes through zero; it is thus possible to operate the hydrogen maser at a temperature such that the wall shift vanishes and to select this temperature by the deformable bulb technique. Recent experiments with spin-polarized hydrogen atoms confined to a low temperature chamber covered with a helium film [68-71] suggest the possibilities of such confining chambers with atomic hydrogen masers. With these new methods, absolute accuracy better than 1 part in $10^{13}$ should be attained. Stabilities better than 1 part in $10^{15}$ have been achieved [72].

Although the hydrogen maser is the most stable atomic maser over long periods of time, Novick, Vanier, and others [35] have developed a high-power optically pumped atomic $\mathrm{Rb}^{85}$ maser whose relatively high output power is useful because it provides good short-term stability over periods of 10 seconds or less.

Hydrogen masers have been extensively used in long baseline interferometry in radio astronomy. Vessot and his associates [73] have used a hydrogen maser in a high altitude rocket to confirm the relativistic gravitational red shift to an accuracy of $0.007 \%$.

\section{Lasers}

Townes and Schawlow [30] pointed out that masers could be produced at infrared and optical frequencies. The first optical maser or laser was successfully made from ruby by Maiman [74]. Subsequently there was a great burst of activity in this field and lasers were made of a wide variety of materials, some of which 
permitted operation at high pulsed power. From the point of view of frequency control the laser using a helium-neon gas mixture developed by Javan [75] and his associates was the first one of interest as a time standard because of its potential stability.

As absolute time standards, most early lasers suffered from the fact that the output frequency was primarily determined by the distance between two mirrors since the first-order Doppler broadening of the atomic or molecular resonance exceeds the resonance width of the interferometer. This characteristic contrasts with a microwave maser where the frequency is determined primarily by the atomic transition with only a relatively small amount of pulling from mistuning of the microwave cavity. However, various methods for diminishing the firstorder Doppler spread and thereby for determining the laser frequency more by atomic or molecular properties have been developed. These methods usually depend upon nonlinear effects.

One method that has been particularly effective is laser-saturated absorption spectroscopy developed by J. L. Hall [76,77], Schawlow [78], Hansch [78], and others [79,80,81-84]. In such a device, laser light is passed in opposite directions through, say, a $\mathrm{CH}_{4}$ or $\mathrm{I}_{2}$ absorption cell. There is a minimum of absorption at a frequency corresponding to no first-order Doppler shift, since stationary molecules absorbing at that frequency absorb the light from both directions equally well and hence are more readily saturated than are the moving molecules which respond at most to light from a single direction. A schematic view of a laser-saturated absorption device is shown in figure 14 . An absorption cell containing methane is included in the optical path between the two mirrors of a heliumneon laser so the methane molecules are subjected to two opposite beams of light. Stabilities of a few parts in $10^{14}$ have been achieved by Hall and others [76] with $3.39 \mu \mathrm{m}$ He-Ne laser-saturated absorption in $\mathrm{CH}_{4}$ but the reproducibility was only about 1 in $10^{11}$. Although this technique markedly reduces first-order Doppler broadening, it does not automatically remove all shifts associated with molecular recoil. Also power shifts and second-order Doppler effects remain. A combination of saturated absorption with an atomic beam of calcium has recently given encouraging results [85].

Double-resonance $[80,86,87]$ and two-photon

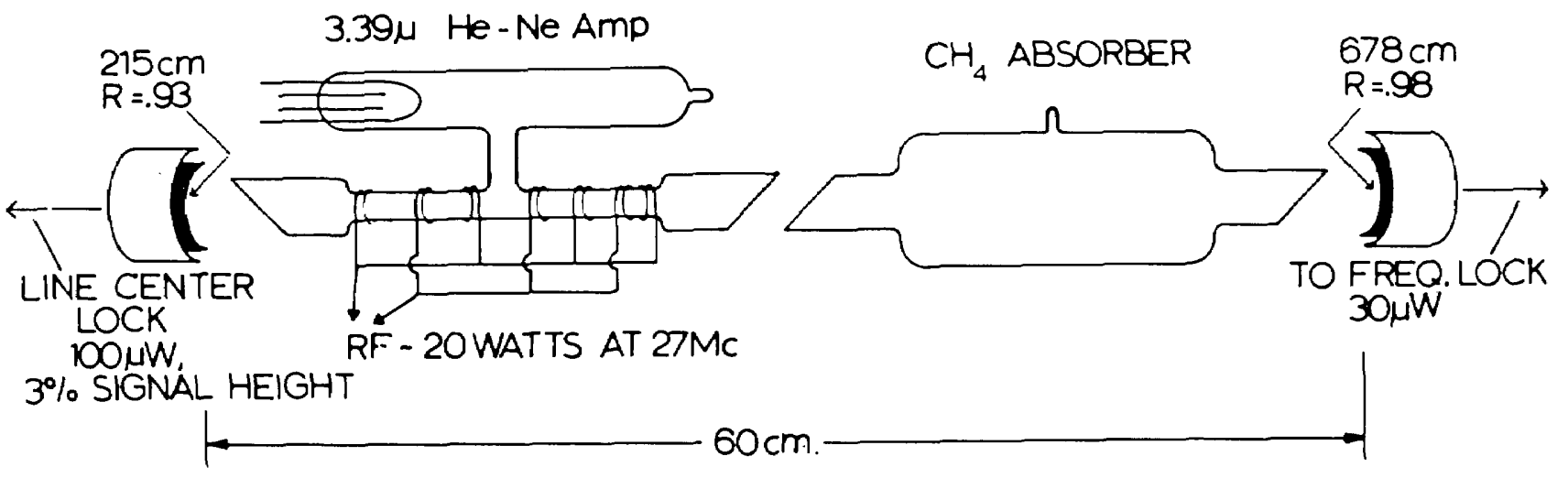

$$
\begin{aligned}
& \left(\Delta v_{H W H M}\right)^{2}=\left\{\left[70 \mathrm{kc}+(16 \mathrm{kc} / \mathrm{m} \text { Torr }) \times P_{\mathrm{CH}_{4}}\right]^{2}+\left[.5 \mathrm{kc} / \mathrm{uW} \times \mathrm{P}_{\text {ext. }}\right]^{2}\right\} \\
& \text { so } \Delta v_{H W H M}-240 \mathrm{kc} \text { at } 300^{\circ} \mathrm{K}, 10 \text { milli Torr \& } 100 \mathrm{uW} \\
& \mathrm{s} / \mathrm{N}(1 \mathrm{sec})-\frac{3 \mathrm{uW} \mathrm{signal}}{1.0 \times \bar{O}^{11} \mathrm{~W} \text { noise }}=3 \times 10^{5}(=109 \mathrm{Db} !) \\
& \delta f=2.3 \frac{\Delta v_{H W H M}}{\mathrm{~S} / \mathrm{N}}=1.9 \mathrm{~Hz} \mathrm{RMS} \\
& \delta f_{/ \mathrm{f}}=2 \times 10^{-14} \times \frac{1}{\sqrt{T_{(\mathrm{sec})}}}
\end{aligned}
$$

Figure 14-Laser-saturated methane frequency reference [76]. 
Doppler-free absorption spectroscopy [88] eliminated first-order Doppler broadening by requiring the absorption of two photons. If these photons come from opposite directions and are at different frequencies appropriate to an intermediate real energy level, the different first-order Doppler shifts would prevent simultaneous absorption of both photons except for the absorption by molecules moving with approximately zero velocity along the direction of the laser beam, since for these molecules the first-order Doppler shifts are approximately zero. Two-photon Doppler-free absorption spectroscopy is particularly effective when the two photons moving in opposite directions are at the same frequency, even though in this case the intermediate transition is to a virtual level since it is unlikely that a real level will fall exactly halfway between the initial and final states. Since the Doppler shift in one direction is equal and opposite to that in the opposite direction, the sum of the two frequencies is independent of the molecular velocity, so molecules at all velocities can contribute to the two-photon Doppler-free spectrum. Since the two photons move in opposite directions with equal momentum, there is no recoil of the molecule and hence no Doppler or recoil broadening. High laser power levels, however, may be required so power shifts may be a problem but they can be reduced with a suitable experimental arrangement. In common with most other methods, the second-order Doppler shift is not eliminated in two-photon spectroscopy.

A major advance in recent years has been the development of frequency multiplying techniques in the optical region by Javan [75]. Evenson and his associates [81,91], Hall and Borde [77] and others. With these techniques it is possible to compare laser frequency standards with the cesium beam standard used in the definition of the second. With such devices, both the frequency and the wavelength of $\mathrm{CH}_{4}$ (and $\mathrm{CH}_{2}$ ) stabilized lasers have been measured and thereby a precision value for the velocity of light of $299792458.3 \pm 1.2 \mathrm{~m} / \mathrm{s}$ has been obtained $[76,89,90]$. This work has now become so accurate that it is expected soon to become the basis for the definition of the meter. The Advisory Committee to the General Conference on Weights and Measures has recommended for the new definition of the meter that "the meter is the length equal to the distance travelled in a time interval $1 / 299,792,458$ of a second by plane electromagnetic waves in a vacuum." When this recommendation is adopted the unit of length will be defined in terms of the second, which in turn is determined by atomic clocks. The velocity of light will cease to be a measurable quantity and instead will be determined by definition.

\section{Trapped Ions}

Dehmelt [91] in 1959 first used electromagnetic ion traps in radio-frequency resonance studies. The intrinsic width of the resonances as determined by the uncertainty principle can be very narrow since the ions are retained in the apparatus for very long periods of time. However, until recently these devices have suffered from the relatively high velocity of the ions in the trap (approximately of $1 \mathrm{eV}$ of kinetic energy) with the correspondingly larger broadening due to the second-order Doppler shift. Initial efforts by Dehmelt et al. [92] to diminish this were only partially successful and trapped-ion devices have not as yet provided frequencies as stable as those of the best alternative frequency standards. However, as will be discussed in the section on Doppler broadening, Wineland and Dehmelt [93] have proposed an ingenious technique for resonant radiation cooling of trapped ions and have shown $[94,95]$ that spectacular line narrowing can be achieved. The resonance widths achieved correspond to temperatures below $50 \mathrm{mK}$ $[94,95]$. If this technique is fully successful, trapped-ion resonance devices should become highly promising frequency standards, although their stability is degraded by the low signal-to-noise ratio which results from the space charge limitation on the number of ions that can be studied simultaneously. Ion recoil ordinarily causes no difficulty since the recoil momentum is absorbed by the trapping field.

\section{Superconducting Cavities}

High-stability superconducting cavity oscillators have recently been made by Stein and others [96] at Stanford University. Although such oscillators do not strictly come within the scope of this report, their stability, especially for short times, is sufficiently great that they should be discussed here at least briefly even though they are not suitable as absolute standards since the frequency depends on cavity dimensions instead of a characteristic atomic or molecular transition frequency. A schematic view of such a superconducting cavity is shown in figure 15 . Stabilities of the order of $10^{-15}$ have been attained over 100 second intervals or more. Since short-term stability increases with oscillator power and since superconducting cavities can be operated at a 


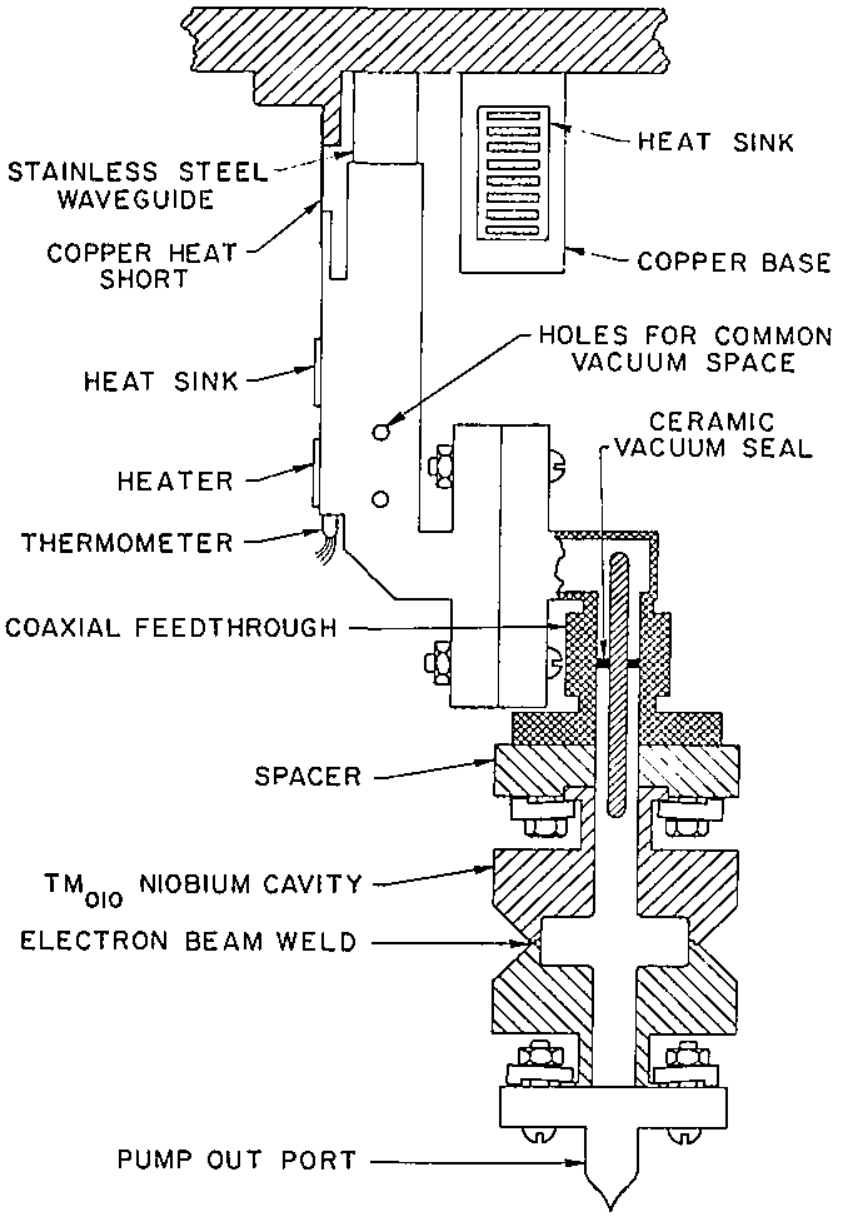

Figure 15-View of superconducting cavity showing the mounting [20].

relatively high power level, they have particularly favorable short-term stability.

\section{Doppler Broadening}

The atoms or molecules in atomic and molecular frequency standards are in thermal motion and hence subject to both first- and second-order Doppler shifts or broadening. The first-order Doppler shift-the familiar increase in the frequency received from an approaching radiation source-is proportional to $\mathrm{v} / \mathrm{c} \sim 3 \times 10^{-7}$ so any competitive frequency standard must provide a means for eliminating the first-order Doppler shift. Consequently, the essential features of the different frequency standards can most simply be given by describing the way that each one eliminates both frequency shifts and resonance broadening from first-order Doppler shifts.

In cesium and molecular beam devices the first- order Doppler shift is eliminated by the use of two separated oscillatory fields of coherent radiation of the same phase. In the hydrogen maser, the first-order Doppler shift is eliminated by confining the hydrogen atoms to a small volume which is traversed many times during the radiation process of each atom so the velocity averages to zero. In trapped-ion spectroscopy, the first-order Doppler shift is eliminated for the same reason. With laser-saturated molecular absorption devices, double-resonance spectroscopy, and two-photon spectroscopy, the firstorder Doppler shift is removed by the requirements of the absorption of two or more photons moving in opposite directions, as discussed in the sections on these devices.

However, even after the first-order Doppler shift is eliminated, there remains in atomic and molecular oscillators a second-order Doppler shift whose magnitude is of the order $(\mathrm{v} / \mathrm{c})^{2} \sim 10^{-13}$. If much progress is to occur beyond the present accuracy of a few parts in $10^{13}$, means must be found to reduce the magnitude of the second-order Doppler shifts, i.e., to reduce the velocities. New possible techniques for reducing the magnitudes of the velocities have been proposed by Hansch and Schawlow [97] and by Wineland and Dehmelt [93]. The basic idea is to cool, say, trapped ions by shining on them intense laser light at a frequency slightly below the resonance frequency. This light can be absorbed by an ion whose motion provides the appropriate first-order Doppler shift. The subsequent emission, however, is in all directions and hence on the average at the normal resonance frequency. By conservation of energy the ion must therefore lose kinetic energy. In this fashion the trapped ions can be cooled by many successive absorptions and reemissions. Dehmelt [94] Wineland [95] and their associates have already demonstrated impressive line narrowing by the laser cooling technique. It will be of great interest during the coming years to see if these techniques for reducing the second-order Doppler width lead to marked increases in the accuracy of clocks and frequency standards.

\section{Accuracy, Reproducibility, and Stability}

In discussions of time and frequency standards it is necessary to distinguish between three different but related properties of the standards: accuracy, reproducibility, and stability. Accuracy measures the degree to which a standard independently agrees with the value specified in the definition of the unit of time. 
Reproducibility is a measure of the extent to which properly adjusted independent devices of the same design agree. Stability is a measure of the degree to which the same device gives the same result in successive intervals of time. The stability is conventionally measured by the parameter $\sigma_{\boldsymbol{r}}(\tau)$ which is the square root of the two-sample Allan variance [98] for adjacent samples which in turn is one-half of the mean square of the fractional differsnces of the frequencies measured in adjacent intervals of time duration $\tau$.

For different applications, different characteristics are the most relevant. Thus, for absolute standards of frequency, the accuracy is the most important property. On the other hand, for many measurements, such as long baseline interferometry in radio astronomy, stability is of primary concern.

The stability parameter $\sigma_{y}(\tau)$ is plotted as a function of the time interval $\tau$ for a number of different kinds of oscillators in figure 16.

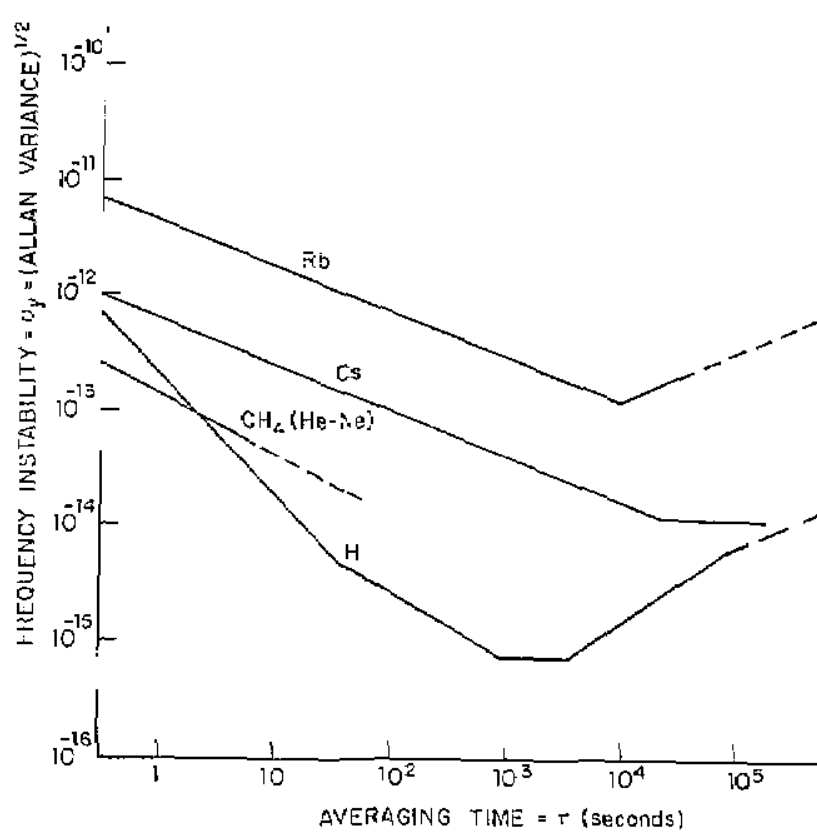

Figure 16-Stabilities of various atomic clocks $[99,96,36,72]$.

The need for accurate and stable timing has been recognized for many centuries and the development of better clocks has been vigorously pursued throughout that time. However, the truly spectacular advances in that field have occurred only in the past few decades, as is illustrated in figure 17. which shows the development of the accuraey of timing through history.

\section{Future Prospects}

Although atomic and molecular frequency and time standards have been a reality for a number of years, developments are occurring at a relatively rapid rate. As a result it is impossible to forecast reliably the future developments that will lead to the most major subsequent advances. However, a number of prospective developments for the different devices are included in the above discussions of these devices. For highest stability and reproducibility the most promising of these prospects are probably the following: (1) Further improvements on the existing atomic and molecular beam methods such as the widely used cesium frequency standard, including better velocity definition to reduce uncertainties due to second-order Doppler shifts and including possible new molecules with higher frequency resonances. (2) Hydrogen maser improvements including new confinement surfaces such as low temperature helium or neon films and combinations of the deformable bulb techniques with either the large box maser or operation at a temperature where the wall shift vanishes. The use of electronic cavity tuning provides increased stability, and interesting studies have been undertaken at the National Bureau of Standards and elsewhere of the use of atomic hydrogen as a passively operating frequency standard [99]. (3) Improved stored-ion devices, especially the new techniques $[93,94,95]$ for cooling the trapped ions to markedly diminish the first- and second-order Doppler broadening. Development of monoion oscillators [85]. (4) The use of lasers especially when combined with nonlinear spectroscopy techniques which eliminate first-order Doppler broadening, such as saturated molecular absorption and especially two-photon Doppler-free spectroscopy; the use of laser cooling techniques [97]. (5) The reduction of the second-order Doppler broadening for any of the methods by the resonance cooling techniques discussed earlier $[93,97,94,95]$. (6) Improvements in frequency multiplying techniques to connect the microwave and optical regions. (7) Improvements in superconducting cavity oscillators. (8) Combinations of various techniques such as saturated absorption laser spectroscopy with atomic beams or use of a superconducting cavity as a slave oscillator for an atomic resonance device. (9) For durations longer than a month measurements on selected pulsars may provide high stability.

If past precedents are followed, there will in the 


\section{THE ACCURACY OF TIMING THROUGH HISTORY}

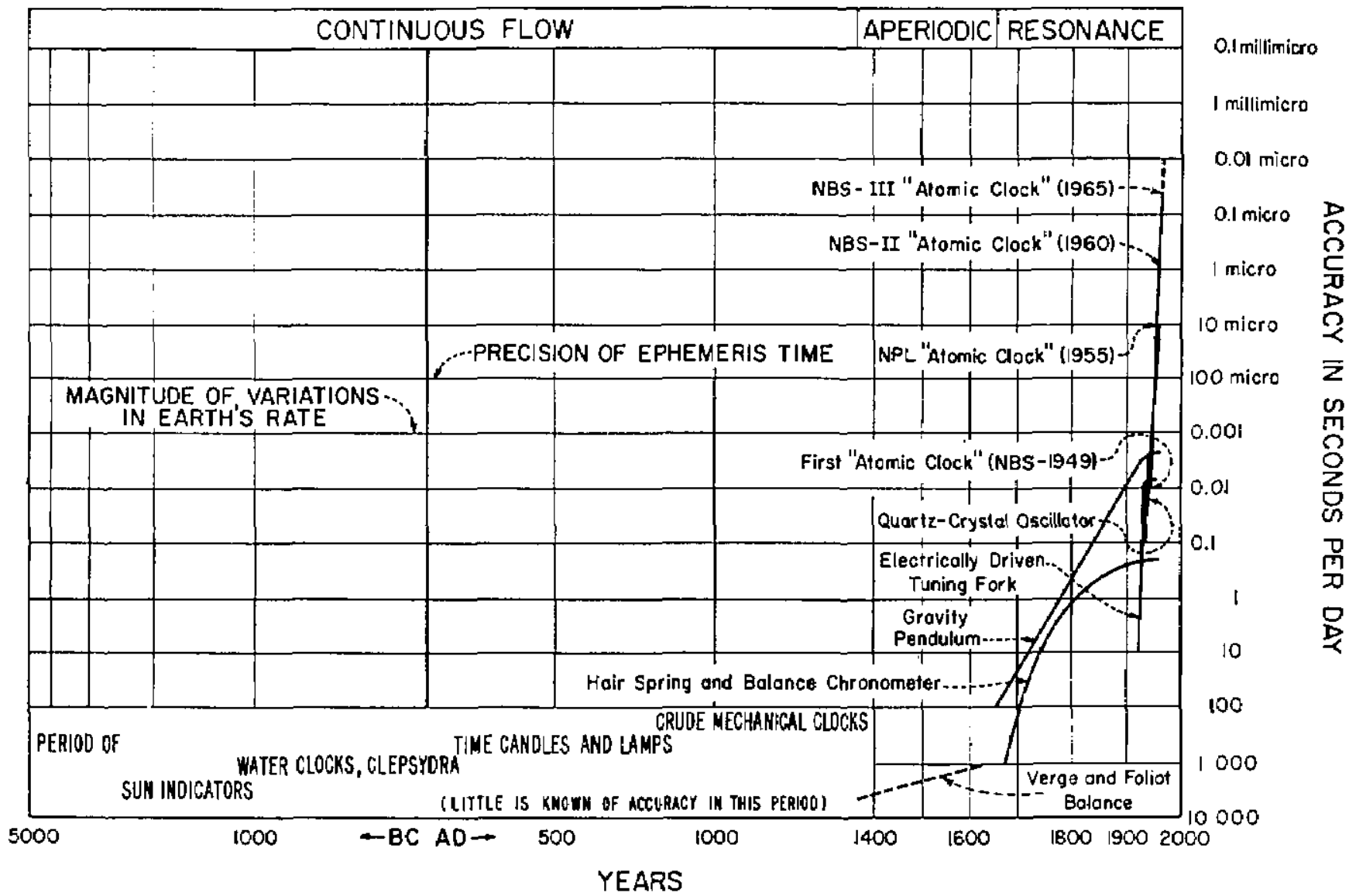

Figure 17-The accuracy of timing through history [25].

future also be many unexpected new ideas and developments that drastically improve existing techniques or lead to totally new kinds of highly stable clocks.

\section{References}

[1] Dunoyer, L. Comptes. Rendu 152, 594 (1911) and L. Radium 8. 142 (1911).

[2] Stern, O. Z. Phys. 39, 751 (1926); Knauer, F. and Stern, O., Z. Phys. 39, 764 and 780 (1926).

[3] Darwin, C. Proc. R. Soc. 117, 258 (1927).

[4] Phipps, T. E.; Stern, O. Z. Phys. 73, 185 (1931).

[5] Guttinger, P. Z. Phys. 73, 169 (1931).

[6] Majorana, E. Nuovo Cimento 9, 43 (1932).

[7] Frisch, R. O.; Segre, E. Z. Phys. 80, 610 (1933).

[8] Rabi, I. l. Phys. Rev. 49, 324 (1936).

[9] Motz, L.; Rose, M. Phys. Rev. 50, 348 (1936).

[10] Rabi, I. I. Phys. Rev. 51, 652 (1937).
[11] Schwinger, J. Phys. Rev. 51, 645 (1937).

[12] Rabi refers to the visit of Gorter in a footnote to the first paper on the molecular beam magnetic resonance method [10] and Gorter 29 years later published an article giving his own somewhat different recollections of the same visit [Physics Today 20, 76 (Jan. 1967).]

[13] Gorter, C. 3. Physica 3, 503 and 995 (1936).

[14] Rabi, I. I.; Zacharias, J. R.; Milman, S.; Kusch, P. Phys. Rev. 53, 318 (1938) and 55, 526 (1939).

[15] Kellogg, J. M. B.; Rabi, I. I.; Ramsey, N. F.; Zacharias, J. R. Phys. Rev. 55, 729 (1939); 56728 (1939); and 57, 677 (1940).

[16] Rabi, I. I. Phys. Rev, 67, 199 (1945),

[17] Kusch, P.; Millman, S.; Rabi, I. I. Phys. Rev. 57, 765 (1940).

[18] Millman, S.; Kusch, P. Phys. Rev. 57, 438 (1940).

[19] Kusch, P.; Taub, H. Phys. Rev. 75, 1477 (1949).

[20] Ramsey, N. F. Phys. Rev. 76, 966 (1949); Molecular Beams, New York; Oxford, 1956 (1959); and IEEE Trans. Instrum. Meas. IM-21, 90 (1972).

[21] Ramsey, N. F.; Silsbee, H. B. Phys. Rev, 84, 506 (1951). 
[22] Lundeen, S. R.; Jessop P. E; Pjpkin, F. M. Phys. Rev. Lett 34, 377 and 1368 (1975).

[23] Kolsky, H. G.; Phipps, T. E.; Ramsey, N. F.; Silsbee, H. B. Phys. Rev. 80, 483 (1950).

[24] Sherwood, J. E.; Lyons, H.; McCracken, R. H.; Kusch, P. Bull. Am. Phys. Soc. 27, No. 1, 43 (1952).

[25] Lyons, H. Ann. N.Y. Acad. Sci. 55, 831 (1952) and Sci. Am. 196, 71 (Feb. 1957).

[26] Zacharias, J. R. private communication and Phys. Rev. 94, 751 (1954). (R. Weiss and $R$. Vessot were associated with Zacharias in the experimental work on the "Fountain" experiment.)

[27] Zacharias, J. R.; Yates, J. G.; Haun, R. D. M.I.T., Res. Lab. Electron., Cambridge, MA, Quart. Prog. Rep. 30, Jan. 1955, and "An Atomic Frequency Standard," Proc. IRE (Abstract) 43, 364 (Mar. 1955).

[28] Basov, N. G.; Prokhorov, A. M. Zh. Eksp. Teor. Fiz. 27, 431 (1954) and 28, 249 (1955); or JETP L.et. 1, 184 (1955).

[29] Bloembergen, N. Phys. Rev, 104, 324 (1956).

[30] Schawlow, A. L.; Townes, C. H. Phys. Rev, 112, 1940 (1958).

[31] Baird, J. K.; Miller, P. D.; Dress, W.; Ramsey, N. F. Phys. Rev. 179, 1285 (1969).

[32] Essen, L.; Parry, V. L. Nature 176, 280, 284 (1955).

[33] Reder, F. H. "Atomic Clocks and Their Applications," USASRDL Tech. Rep. 2230 (AD 265452), 1961.

[34] Ramsey, N. F. Phys. Rev. 100, 1191 (1954); 169, 822 (1958); J. Phys. (Paris) 19, 809 (1958); I. Esterman, Editor, Recent Research in Molecular Beams, 107, Academic Press, New York, 1959.

[35] Proc. Frequency Control Symposia 1955-1982; IEEE Trans. Instrum. Meas. IM-13 (1964); IEEE Trans. Instrum. Meas. IM-15 (1966); IEEE Trans. Instrum. Meas. IM-19 (1970); also IEEE Trans. Quantum Electron. QE-5 (1969). R. E. Beehler, Ann. Freq. Control Symp. 25, (1971).

[36] Hellwig, H.; Evenson, K. M.; Wineland, D. J. Physics Today 31, 23 (Dec. 1978).

[37] Bonanomi, J. Quantum Electronics III, Columbia Liniv. Press, New York, 1964.

[38] Cleeton, C. E.; Williams, N. H. Phys. Rev. 45, 234 (1934).

[39] Concon, E. V.; Odishaw, H. Handbook of Physics, McGraw. Hill, New York, 1967.

[40] Townes, C. H.; Schawlow, A. L. Microwave Spectroscopy, McGraw-Hill, New York, 1955.

[41] Townes, C. H. J. Appl. Phys. 22, 1365 (1951).

[42] Dicke, R. H. Phys. Rev. 89, 472 (1953).

[43] Bitter, F. Phys. Rev. 76, 833 (1949), and Pryee, M. H. T. Phys. Rev. 77, 136 (1950).

[44] Brossel, J.; Kastler, A. C. R. Acad. Sci. (Paris) 229, 1213 (1949).

[45] Kastler, A. J. Phys. (Paris) 11, 225 (1950), and J. Opt. Soc. Am. 47, 460 (1957).

[46] Bouchiat, M. A.; Brossel, J. Phys. Rev. 147, 41 (1966).

[47] Goldenberg, H. M.; Kleppner, D.; Ramsey, N. F. Phys. Rev. Lett. 8, 361 (1960).

[48] Pound, R. V.; Purcell, E. M.; Ramsey, N. F. Phys. Rev. 81, $156,278,279$ (1951) and 103, 20 (1956).

[49] Gordon, J. P.; Zeiger, H. Z.; Townes, C. H. Columbia Rad. Lab. Prog. Rep., Dec. 1951; J. Commun. Eng. Japan 36, 650 (1953); and Phys. Rev. 95, 282 (1954); also Phys. Rev. 99, 1264 (1955).

[50] Weber, J. "Amplification of Microwave Radiation by Substances Not in Thermal Equilibrium," Trans. IRE Electron Devices ED.3, 1-4 (June 1953).

[51] Kleppner, D.; Ramsey, N. F.; Fjelstadt, P. Phys, Rev, Lett. 1, 232 (1958)
[52] Teflon is a registered trademark of the duPont Corporation for certain fluorohydrocarbons. The FEP Teflon was found to provide a more uniform coating for the hydrogen maser storage vessels.

[53] Goldenberg, H. M.; Kleppner, D.; Ramsey, N. F. Phys. Rev. 123, 530 (1961).

[54] Paraflint is a registered trademark of Moore and Munger of New York City for certain long chain paraffins.

[55] Dri-Film is a registered trademark of the General Electric Co. for dimethyldichlorosilane.

[56] Kleppner, D.; Goldenberg, H. M.; Ramsey, N. F. Phys. Rev. 126, 603 (1962); Berg, H. C.; Kleppner, D. Rev. Sci. Instrum. 33, 238 (1962).

[57] Bender, P. L. Phys. Rev. 132, 2154 (1963).

[58] Crampton, S. B. Phys. Rev. 158, 57 (1967).

[59] Crampton, S. B.; Duvivier, J. A.; Read, G. S.; Williams, E. R. Phys. Rev. A5, 1952 (1972), Crampton, S. B.; Wong, H. T. M. Phys. Rev. A12, 1305 (1975); Bull. Am. Phys. 18, 709 (1973) and 19, 83 (1974).

[60] Kleppner, D.; Berg, H. C.; Crampton, S. B.; Ramsey, N. F.; Vessot, R. F. C.; Peters, H. E.; Vanier, J. Phys. Rev. 138, A972 (1965).

[61] Audoin, C. Rev. Phys. Appl. 1, 2 (1966) and 2, 309 (1967); Phys. Lett. 28A, 373 (1968); Audoin, C.; Desaintfuscien, M.; Petit, P.; Schermann, J. P. Nucl. Instrum. Methods 69, 1 (1969); Design of a double focalization in a hydrogen maser, IEEE Trans. Instrum. Meas. IM-17, 351-358 (Dec. 1968). (This work utilizes a useful double-focusing method to eliminate the undesired $F=1 \mathrm{~m} \mathrm{~F}=1$ state from the focused beam.; Electron. Lett. 5, No. 13 (1969); C. R. Acad. Sci. (Paris) 264, 698 (1967) and 270, 906 (1970); Double-resonance method for determination of level populations, IEEE J. Quantum Electron. QE-5, 431-434 (Sept. 1969); Haroche, S,; Cohen-Tanoudjt, C.; Audoin, C.; Schermann, J. P. Phys. Rev. Lett. 24, 861 (1970).

[62] Laboratories that have engaged in hydrogen maser studies include Harvard University, Massachusetts Institute of Technology, Bomac Laboratories, Varian Associates, Hewlett-Packard, the National Bureau of Standards, Goddard Space Flight Center, the Jet Propulsion Laboratory, U.S. Electronics Command, Hugher Research Laboratory. Laboratoire de l'Orloge Atomique, Orsay (France), PTB (Braunschweig, Germany), the National Research Council and Laval Lniversity (Canada), R. R. L. (Tokyo, Japan), LSRH (Neuchatel, Switzerland), and the Lebedev Institute (Moscow, L.S.S.R.), ASUAG, Efratom, Applied Physics Laboratory, Moscow State University, Shanghai and Shianxi Observatory and the National Institute of Metrology in China.

[63] Uzgiris, E.; Ramsey, N. F. Phys. Rev. A1, 429 (1970),

[64] Brenner, D. J. Appl. Phys. 41, 2942 (1970).

[65] Debely, P. E.; Rev. Sci. Instrum. 41, 1290 (1970).

[66] Reinhardt, V.; Lavanceau, J. Proc. Annu. Symp. on Freq. Control 28, 379 (1974).

[67] Zitzewitz, P. W; Ramsey, N. F. Phys. Rev. A3, 51 (1971).

[68] Silvera, I. F.; Walraven, J. T. M.; Mathey, A. P. M. Phys. Rev. Lett. 44, 164 (1980) and 45, 449 (1980).

[69] Cline, R. W.; Smith, D. A.; Greytak, T. J.; Kleppner, D. Phys. Rev. Lett. 45, 2117 (1980).

[70] Crampton, S. B.; Greytak, T. J.; Kleppner, D. Phillips, W. D.; Smith, D. A.; Weinrib, A. Phys. Rev. Lett. 42, 1039 (1979).

[71] Hardy, W. N.; Berlinsky, A. J.; Whitehead, L. A. Phys. Rev. Lett. 42, 1042 (1979).

[72] Sydnor, R. L.; Kuhorle, P. F.; Kirk, A.; Endsley, E. F.; Meyer, R. F.; Zygielbaum, A. I. Final Report Hydrogen 
Maser Comparison Test, Jet Propulsion Laboratory (1982).

[73] Vessot, R. F. C. Levine, M. W.; Matison, E. M.; Blomberg, E. L.; Hoffman, T. E.; Nystrom, G. V. Farrel, B. F.; Decher, R.; Eby, P. B.; Baugher, C. R.; Watts, J. W.; Teuber, D. L.; Wills, F. D. Phys. Rev. Lett. 26, 208 (1980).

[74] Mainman, T. H. Nature 187, 493 (1960).

[75] Javan, A.; Bennett, W.; Herriott, D. R. Phys. Rev. Lett. 6, 106 (1961); Hocker, L. O.; Small, J. G.; Javan, A. Phys. Rev. Lett. 29A, 321 (1969).

[76] Barger, R. L.; Hall, J. L. Phys. Rev. Lett. 22, 4 (1969); Appl. Phys. Lett. 22, 196 (1973); Atomic Masers and Fundamental Constants 5, 322 (1976) (Plenum Press).

[77] Hall, J. L.; Borde, C. Phys. Rev. Lett. 30, 1101 (1973).

[78] Hansch, T. W.; Levenson, M. D.; Schawlow, A. L. Phys. Rev. Lett. 26, 946 (1971).

[79] Evenson, K. M. et al., Phys. Rev. Lett. 29, 1346 (1972).

[80] Brewer, R. G. Science 178, 247 (1972).

[81] Mungall, A. S. et al., Metrologia 17, 123 (1981).

[82] Becker, G. Metrologia 18, 17 (1982).

[83] Arditi, M. Metrologia 18, 59 (1982).

[84] de Marchi, A. Metrologia 18, 103 (1982).

[85] Barger, R. Z.; English, T. C.; West, J. B. Annu. Symp. on Freq. Control 29, 316 (1975) (U.S. Army Signal Corps. Ft. Monmouth, NJ).

[86] Schlossberg, H. R; Javan, A. Phys. Rev. Lett. 17, 1242 (1966).

[87] Hansch, T. W.; Shahin, I. S.; Schawlow, A. L. Phys. Rev. Lett. 27, 707 (1971).

[88] Visikenko, L. S.; Chebotaev, V. P.; Shishaev, A. V. JETP Lett. 12, 113 (1970); Pritchard, D.; Apt, J.; Ducas, T. W. Phys. Rev. 32, 641 (1974); Levenson, M. D.; Bloembergen,
N. Phys. Rev. Lett. 32, 645 (1974); Birahan, F.; Cagnac, B.; Grynberg, G. Phys. Rev. Lett. 32, 643 (1974); Hansch, T. W., et al., Opt. Comm. 11, 50 (1974).

[89] Evenson, K. M.; Wells, J. S.; Peterson, F. R.; Danielson, B. L.; Day, G. W. Appl. Phys. Lett. 22, 192 (1973) and 20, 296 (1972); Phys. Rev. Lett. 31, 573 (1973).

[90] Jolliffe, B. W.; Rowley, W. R. C.; Shotton, K. C.; Wallard, A. J.; Woods, P. Z. Nature 251, 46 (1974).

[91] Dehmelt, H. G. Phys. Rev, 109, 381 (1959); Advances in Atomic Molecular Physics 3, 53 (1967) and 5, 109 (1959).

[92] Dehmelt, H. G.; Major, F.; Fortson, E. N.; Schuessler, H. A. Phys. Rev. Lett. 8, 213 (1967); Phys. Rev. 170, 91 (1968) and 187, 5 (1969).

[93] Wineland, D.; Dehmelt, H. Bull. Am. Phys. Soc. 18, 1521 (1973) and 20,60, 61, 637 (1975).

[94] Neuhauser, W.; Hohenstatt, M.; Toschek, P. E.; Dehmelt, H. Phys. Rev. A22, 1137 (1980).

[95] Wineland, D. J.; Itano, W. M.; Bergquists, J. C.; Dryllinger, R. E. Phys. Lett. 82A, 75 (1981); Optics Letters 5, 245 (1980) and Phys. Rev. A20, 152 (1980).

[96] Stein, S. R.; Turneauve, J. P. Proc. Annu. Symp. Freq. Control 27, 414 (1973), and HPL 741, Stanford High Energy Physics Laboratory, Stanford, CA.

[97] Hansch, T. W.; Schawlow, A. L. Opt. Commun. Netherlands 13, 68 (1975) and review by U.S. Letokhov, Comments on At. Mol. Phys. 6, 119 (1977).

[98] Allan, D. W. Proc. IEEE 54, 221 (1966).

[99] Hellwig, H. W. Proc. IEEE 63, 212 (1975); Metrologia 6, 56 (1970); and NBS Tech. Note 616, 1 (1972) and 662, 1 (1975). 